\title{
Basel - III Framework on Liquidity Standards - A Case Study on South Indian Bank Ltd.."
}

Subramoniam K.*

Assistant Professor, Saintgits Institute of Management, Kottayam, Kerala - 686532, India

\section{Abstract}

A bank is said to have adequate liquidity when it can raise sufficient funds both by increasing liabilities (deposits) and by realizing assets promptly. Bank's liquidity management, therefore, is the process of generating funds to meet contractual obligations like new loan demand, existing loan commitment and deposit withdrawals at reasonable prices at all times. The main forms of liquidity risks are 'Funding Risk', 'Time Risk' and 'Call Risk'. Measuring and managing liquidity risk are one of the most vital activities of commercial banks. This case study brings out the procedure given by Reserve Bank in the lines of Basel III framework and the liquidity management practices of South Indian Bank. While analysing the liquidity in Flow approach and Stock approach it is observed that there is a better liquidity for the Bank to meet obligations in some time buckets and in some, the position needs improvement.

Keywords: Liquidity Risk, Basel III Framework, Funding Risk, Time Risk, Call Risk, Stock Approach, Flow Approach, Time Bucket

\section{Introduction}

South Indian Bank Limited (SIB) (BSE: 532218, NSE: SOUTH BANK) is a private sector bank headquartered at Thrissur in Kerala, India. South Indian Bank has 831 branches, 4 service branches 33 extension counters and 20 regional offices spread across more than 26 states and three union territories in India. It has set up 1269 ATMs and 4 Bulk Note Acceptor/Cash Deposit Machines all over India. One of the earliest banks in South India, "South Indian Bank" came into being existence during the Swadeshi movement. South Indian Bank was registered as a private Limited Company under the companies Act of 1913 and commenced business on $29^{\text {th }}$ January, 1929 at Round South, Thrissur. The establishment of the Bank was the fulfillment of the dreams of a group of enterprising men who joined together at Thrissur, a major town (now known as the Cultural Capital of Kerala), in the erstwhile State of Cochin.
The composition of the Board of Directors is governed by the Banking Regulation Act, 1949, the Companies Act, 2013, SEBI (Listing Obligations and Disclosure Requirements) Regulations 2015 and the Code of Conduct on Corporate Governance adopted by the Bank. The Board comprises of 10 Directors as on the date of this report, with rich experience and specialized knowledge in various areas of relevance to the Bank, including banking, accountancy, small scale industry, agriculture, and information technology. Excluding the MD \& CEO, all other members of the Board are Non-Executive Directors and Seven Directors out of the total 10 Directors are Independent Directors. Declaration has been obtained from the Independent Directors as required under the RBI Regulations, SEBI (Listing Obligations and Disclosure Requirements) Regulations 2015 and Companies Act, 2013. The remuneration and other benefits paid to MD \& CEO of the Bank and other Non-Executive and Independent

\footnotetext{
*Email: ksubramoniam200420@yahoo.com

\#This is the Revised and modified version of the article, presented in the 6th International Conference on Emerging Trends in in Finance, Accounting and Banking, SDMIMD, August 2017.
} 
Directors during the financial year 2015-16 are disclosed in Corporate Governance Report.

Mr. Sunil Gangadharan is the Chairman and Mr. V. G. Mathew is the CEO and Managing Director of South Indian Bank.

\subsection{Need for Study}

The main purpose behind this study is to understand about the liquidity risk management measures under Basel III. This study helps us to see how SIB manage their risks in an effective way.

\subsection{Objective of the study}

To study the liquidity risk and liquidity risk management measures being followed by South Indian Bank.

The different sections of the paper are given below:

1. Review of Literature

2. Dimensions and Role of Liquidity Risk Management

3. Methodology

4. Data Analysis and Interpretation

5. Findings

6. Conclusion

\section{Review of Literature}

Though the importance of liquidity risk is well recognized, it eludes a comprehensive definition. The term liquidity is used across the market for different purposes, which means that liquidity risk itself is defined differently and depends very much on the context in which it is used. Liquidity risk is sometimes referred to as inability of the bank to convert an asset into cash at a reasonable time at a specific price. The cash position of the firm is an important consideration in paying dividends; the grater the cash position and overall liquidity of a company, the greater will be its pay dividends (Pandey, 2010). Literature contains many more such contextual definitions.

Padmalatha. S and Justin P (2010) give the anatomy of liquidity risk in the following model

\section{Anatomy of liquidity risk}

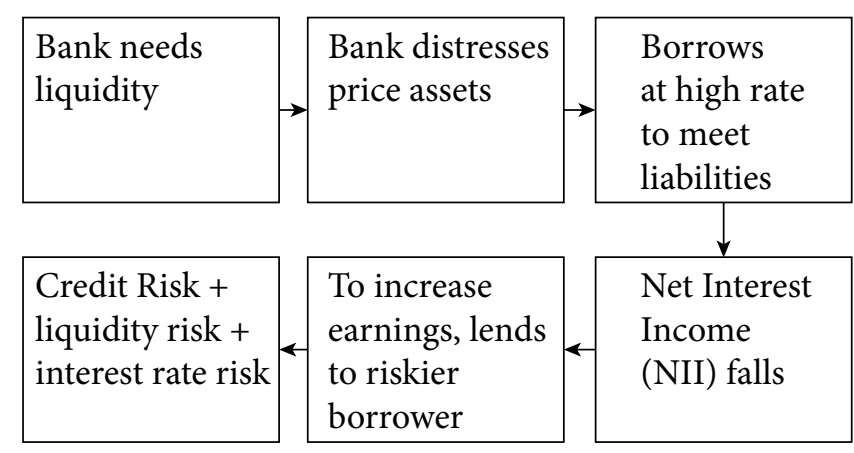

This model brings out the most significant impact of liquidity problems. They can have an adverse impact of the bank's earnings and capital, and, in extreme circumstances, may even lead to the collapse of the bank itself, though the bank may otherwise be solvent. A more dangerous consequence is that a liquidity crisis in a large bank could give rise to systemic consequences impacting other banks and the country's banking system as a whole. Liquidity problems can also affect the proper functioning of payment systems and other financial markets.

In its paper titled 'Liquidity Risk Management and Supervisory Challenges', the Basel Committee (2008) pointed out that

a. Most of the banks which exposed themselves to severe liquidity risks did not have the requisite framework to support the risks inherent in individual business lines or products, and, therefore, did not align the risks to the bank's own risk tolerance.

b. Many banks did not account for the 'unlikely' event of a large part of their contingent liabilities having to be funded all at once.

c. To many banks, the kind of severity or duration of the liquidity crisis (as it materialized later) seemed a remote possibility. Hence, these banks did not conduct stress tests to factor market wide liquidity strain and disruptions.

d. Even where contingency funding plans were made, they were not related to results of stress tests, or did not factor in the possible drying up of some potential funding sources.

Subramoniam (2015) made a discussion on following sources of liquidity risk: 
a. A decline in earnings: The income of the bank can come down because of many reasons. The decline in Net Interest Income (NII) is one of the major reasons. The spread between lending rate and deposit rate may come down due to market pressure or RBI directives. Similarly, non-interest income may come down due to offering service at a cheap rate or free of cost to meet the competition in the banking sector.

b. An increase in Non-Performing Assets (NPA): NPA is a double-edged sword; on the one side, the income cannot be recognized from NPAs and on the other side, provision has to be kept. Both this affect profitability. Moreover, the funds blocked in NPAs will cause liquidity problem as they are not readily available for recycling. This results in 'credit squeeze'.

c. Deposit concentration: Some banks rely on bulk deposits from large corporate or government departments which may be withdrawn in short period. Sometimes, it will cause 'liquidity shocks' if banks are not able to mobilize similar deposits.

d. Downgrading by rating agencies: When Moody's downgraded State Bank of India, it caused a shock to the banking sector as a whole. The banks may suffer from 'deposit drain' on such occasions.

e. Expanded business opportunities: When a bank is aiming at horizontal growth through branch expansion, the business opportunities are also growing. Likewise, a growing economy demands more funds, and to meet the competition, banks may have to lend across the sectors. This necessitates liquid funds.

f. Acquisitions: This is one of the sources of liquidity problems as acquisitions require payment of huge cash to the entity acquired.

g. New tax initiatives: This may require cash outflow for meeting tax obligations.

The literature shows the importance and significance of liquidity management.

\subsection{Dimensions and Role of Liquidity Risk Management}

Banks need liquidity to meet deposit withdrawal and to fund loan demands. The variability of loan demands and variability of deposits determine bank's liquidity needs. It represents the ability to accommodate decreases in liability and to fund increases in assets.
Effective liquidity management by bank serves four important functions.

- It demonstrates the market place that the bank is safe and therefore capable of repaying its borrowings.

- It enables bank to meet its prior loan commitments, whether formal or informal.

- It enables bank to avoid the unprofitable sale of assets.

- It lowers the size of the default risk premium which the bank must pay for which funds.

\section{Methodology}

For measuring and managing liquidity risk, two measures are prescribed under Basel III. They are:

1. Flow Approach

2. Stock Approach

Flow Approach: consists the following: Flow method is the basic approach followed by Indian Banks. It is called as gap method of measuring and managing liquidity. It requires the preparation of structural liquidity gap report. In this method net funding requirement is calculated on the basis of residual maturities of assets $\&$ liabilities. These residual maturities represent net cash flows i.e., difference between cash outflow \& cash inflow in future time buckets.

These calculations are based on the past behavior pattern of assets and liabilities as well as off-balance sheet exposures. Cumulative gap is calculated at various time buckets. In case gap is negative bank has to manage the shortfall.

The analysis of net funding requirements involves the construction of a maturity ladder and the calculation of a cumulative net excess or deficit of funds at selected maturity dates.

The RBI guidelines for the gaps are given below:

A Structural Liquidity Statement should be prepared by the Bank and to be submitted to RBI on fortnightly basis on the first and third Wednesday of each month. 
The various time buckets considered for the purpose and the RBI guideline for gaps are given below:

Table 1. Structural Liquidity Statement

\begin{tabular}{|c|c|c|}
\hline SI. No. & Time Buckets & $\begin{array}{l}\text { RBI Guidelines- Negative Gap } \\
\text { should not exceed }\end{array}$ \\
\hline 1 & Day 1 & $5 \%$ of the total outflow \\
\hline 2 & 2-7 days & $10 \%$ of the total outflow \\
\hline 3 & 8-14 days & $15 \%$ of the total outflow \\
\hline 4 & $15-28$ days & $20 \%$ of the total outflow \\
\hline 5 & 29 days and upto 3 months & \multirow{6}{*}{$\begin{array}{l}\text { Each Bank can have its own ALCO/ } \\
\text { Board approved policy regarding } \\
\text { the permissible gaps. SIB tries } \\
\text { to keep positive gap in all time } \\
\text { buckets. }\end{array}$} \\
\hline 6 & Over 3 months upto 6 months & \\
\hline 7 & Over 6 months and upto 1 year & \\
\hline 8 & Over 1 year and upto 3 years & \\
\hline 9 & Over 3years and upto 5 years & \\
\hline 10 & Over 5 years & \\
\hline
\end{tabular}

Stock Approach: is based on the level of assets and liabilities as well as off balance sheet exposures on a particular date. The following ratios are calculated to assess the liquidity position of the bank:

- Ratio of core deposits to total assets: More the ratio, better it is because core deposits are treated to be stable source of liquidity. Core deposits will constitute deposits from the public in the normal course of business.

- Net loans to total deposits ratio: It reflects the ratio of loans to public deposits or core deposits. Total loans in this ratio represent net advances after deduction of provision for loan losses and interest suspense account. Loan is treated to be less liquid asset and therefore lower ratio, better it is.
- Ratio of time deposits to total deposits: Time deposits provide stable level of liquidity and negligible volatility. Therefore, lower the ratio better it is.

- Ratio of volatile liabilities to total assets: Volatile liabilities like market borrowings are to be assessed and compared with the total assets. Higher portion of volatile liabilities cause higher liquidity problems. Therefore lower the ratio is better.

- Ratio of short term liabilities to liquid assets: short term liabilities are required to be redeemed at the earliest. Therefore they will require ready liquid assets to meet the liability. It is expected to be lower in the interest of liquidity Ratio of liquid assets to total assets: higher level of liquid assets in total assets will ensure better liquidity. Therefore higher the ratio is better.

- Ratio of short term liabilities to total assets: Shortterm liabilities may include balances in current account, volatile portion of savings account leaving behind core portion of saving which is constantly maintained. A lower ratio is desirable.

- Ratio of prime assets to total assets: Prime assets may include cash balances with banks and balances with the banks including central bank which can be withdraw at any time without any notice. Higher the ratio is better.

- Ratio of market liabilities to total assets: Market liabilities may include money market borrowings, interbank liabilities payable within a short period. Lower the ratio is better.

For the purpose of this study, data from the various annual reports of SIB are collected covering a period of 2011 to 2016.

\section{Data Analysis and Interpretation}

\section{Flow approach - finding gap from 2011-2016:} Maturity Pattern of key assets and liabilities:

As at $31^{\text {st }}$ March, 2011:

Exhibit 1:

\begin{tabular}{|l|l|l|l|l|l|l|l|l|l|l|}
\hline Particulars 2011 & Day 1 & $\mathbf{2 - 7}$ days & $\begin{array}{l}\mathbf{8 - 1 4} \\
\text { days }\end{array}$ & $\begin{array}{l}\mathbf{1 5 - 2 8} \\
\text { days }\end{array}$ & $\begin{array}{l}\mathbf{2 9} \text { days } \\
\text { and upto } \\
\mathbf{3} \text { months }\end{array}$ & $\begin{array}{l}\text { Over 3 } \\
\text { months } \\
\text { and upto 6 } \\
\text { months }\end{array}$ & $\begin{array}{l}\text { 0ver 6 } \\
\text { months } \\
\text { and upto 1 } \\
\text { year }\end{array}$ & $\begin{array}{l}\text { Over 1 } \\
\text { year and } \\
\text { upto 3 } \\
\text { years }\end{array}$ & $\begin{array}{l}\text { 0ver 3 } \\
\text { year and } \\
\text { upto 5 } \\
\text { years }\end{array}$ & $\begin{array}{l}\text { 0ver 5 } \\
\text { years }\end{array}$ \\
\hline Liabilities & & & & & & & & & & \\
\hline Deposits & 152.32 & $1,171.88$ & 963.1 & 433.84 & $3,639.23$ & $2,865.94$ & $6,584.86$ & $11,214.99$ & 557.06 & $2,137.86$ \\
\hline Borrowings & 24.67 & - & - & - & 0.06 & 0.06 & 0.11 & 65.45 & - & 200 \\
\hline
\end{tabular}




\begin{tabular}{|l|l|l|l|l|l|l|l|l|l|l|l|}
\hline Foreign Currency Liabilities & 44.65 & - & - & 0.36 & 0.04 & 2.17 & 1.74 & - & - \\
\hline Total liabilities & 221.64 & $1,171.88$ & 963.1 & 434.2 & $3,639.33$ & $2,868.17$ & $6,586.71$ & $11,280.44$ & 557.06 & $2,337.86$ \\
\hline Assets & & & & & & & & & & \\
\hline Loans \& Advances & 403.28 & 209.31 & 316.08 & 508.91 & 3029.17 & 2839.51 & 6636.55 & 3149 & 1347.16 & 2049.76 \\
\hline Investments & 7.59 & 455.83 & 284.31 & 100.64 & 1140.51 & 161.68 & 124.2 & 625.14 & 1377.11 & 4646.76 \\
\hline Foreign Currency Assets & 12.99 & - & 37.54 & 0.48 & 17.14 & 91.04 & 56.78 & 216 & - & - \\
\hline Total Assets & 423.86 & 665.14 & 637.93 & 610.03 & 4186.82 & 3092.23 & 6817.53 & 3990.14 & 2724.27 & 6696.52 \\
\hline
\end{tabular}

Source: Annual Report of SIB, March 2011

Maturity Ladder Based on Contractual Maturities 2011:

\begin{tabular}{|c|c|c|c|c|c|c|c|}
\hline $\begin{array}{l}\text { DAY } 1 \\
\text { CASH INFLOWS }\end{array}$ & AMOUNT & CASH OUTFLOWS & & AMOUNT & GAP & RBI TOLERANCE LIMITS & REMARKS \\
\hline Loans and Advances & 403.28 & Deposits & & 152.32 & & \multirow[t]{4}{*}{$\begin{array}{l}\text { Negative Gap should not be } \\
\text { more than } 5 \% \text { of total outflows }\end{array}$} & Gap is positive RBI Compliant \\
\hline Investments & 7.59 & Borrowings & & 24.67 & & & \\
\hline $\begin{array}{l}\text { Foreign Currency } \\
\text { Assets }\end{array}$ & 12.99 & $\begin{array}{l}\text { Foreign Currency } \\
\text { Liabilities }\end{array}$ & & 44.65 & & & \\
\hline Total & 423.86 & & & 221.64 & 202.22 & & \\
\hline $\begin{array}{l}\text { DAY 2-7 } \\
\text { CASH INFLOWS }\end{array}$ & & CASH OUTFLOWS & & & & $\begin{array}{l}\text { Negative Gap should not be } \\
\text { more than } 10 \% \text { of total outflows }\end{array}$ & $\begin{array}{l}\text { Negative Gap is } 43 \% \text { of total } \\
\text { outflow. so it is not RBI Compliant }\end{array}$ \\
\hline Loans and Advances & 209.31 & Deposits & & $1,171.88$ & & & \\
\hline Investments & 455.83 & Borrowings & - & & & & \\
\hline $\begin{array}{l}\text { Foreign Currency } \\
\text { Assets }\end{array}$ & - & $\begin{array}{l}\text { Foreign Currency } \\
\text { Liabilities }\end{array}$ & - & & & -506.74 & \\
\hline Total & 665.14 & & $1,171.88$ & & & & \\
\hline $\begin{array}{l}\text { 8-14 days } \\
\text { CASH INFLOWS }\end{array}$ & & CASH OUTFLOWS & & & & $\begin{array}{l}\text { Negative Gap should not be } \\
\text { more than } 15 \% \text { of total outflows }\end{array}$ & $\begin{array}{l}\text { Negative gap is } 33 \% \text { of the total } \\
\text { cash outflow. Here the bank has } \\
\text { breached RBI tolerance limits }\end{array}$ \\
\hline Loans and Advances & 316.08 & Deposits & 963.1 & & & & \\
\hline Investments & 284.31 & Borrowings & - & & & & \\
\hline $\begin{array}{l}\text { Foreign Currency } \\
\text { Assets }\end{array}$ & 37.54 & $\begin{array}{l}\text { Foreign Currency } \\
\text { Liabilities }\end{array}$ & - & & & & \\
\hline Total & 637.93 & & 963.1 & -325.17 & & & \\
\hline $\begin{array}{l}\text { 15-28days } \\
\text { CASH INFLOWS }\end{array}$ & & CASH OUTFLOWS & & & & & \\
\hline Loans \& Advances & 508.91 & Deposits & & 433.84 & & $\begin{array}{l}\text { Negative Gap should not be } \\
\text { more than } 20 \% \text { of total outflows }\end{array}$ & Gap is positive. RBI Compliant \\
\hline Investments & 100.64 & Borrowings & & - & & & \\
\hline $\begin{array}{l}\text { Foreign Currency } \\
\text { Assets }\end{array}$ & 0.48 & $\begin{array}{l}\text { Foreign Currency } \\
\text { Liabilities }\end{array}$ & & 0.36 & & & \\
\hline Total & 610.03 & Total & & 434.2 & 175.83 & & \\
\hline
\end{tabular}




\begin{tabular}{|c|c|c|c|c|c|c|c|c|}
\hline $\begin{array}{l}29 \text { days and upto } 3 \\
\text { months } \\
\text { CASH INFLOWS }\end{array}$ & & CASH OUTFLOWS & & & & & & \\
\hline Loans \& Advances & 3029.17 & Deposits & & $3,639.23$ & & $\begin{array}{l}\text { Gap is positive. } \\
\text { RBI Compliant }\end{array}$ & & \\
\hline Investments & 1140.51 & Borrowings & & 0.06 & & & & \\
\hline $\begin{array}{l}\text { Foreign Currency } \\
\text { Assets }\end{array}$ & 17.14 & $\begin{array}{l}\text { Foreign Currency } \\
\text { Liabilities }\end{array}$ & & 0.04 & & & & \\
\hline $\begin{array}{l}\text { Foreign Currency } \\
\text { Assets }\end{array}$ & 17.14 & $\begin{array}{l}\text { Foreign Currency } \\
\text { Liabilities }\end{array}$ & & 0.04 & & & & \\
\hline Total & 4186.82 & Total & & $3,639.33$ & 547.49 & & & \\
\hline $\begin{array}{l}\text { Over } 3 \text { months and } \\
\text { upto } 6 \text { months } \\
\text { CASH INFLOWS }\end{array}$ & & CASH OUTFLOWS & & & & & & \\
\hline Loans \& Advances & 2839.51 & Deposits & & $2,865.94$ & & Gap is positive. & & \\
\hline Investments & 161.68 & Borrowings & & 0.06 & & & & \\
\hline $\begin{array}{l}\text { Foreign Currency } \\
\text { Assets }\end{array}$ & 91.04 & $\begin{array}{l}\text { Foreign Currency } \\
\text { Liabilities }\end{array}$ & & 2.17 & & & & \\
\hline Total & 3092.23 & Total & & $2,868.17$ & 224.06 & & & \\
\hline $\begin{array}{l}\text { Over } 6 \text { months and } \\
\text { upto } 1 \text { year } \\
\text { CASH INFLOWS }\end{array}$ & & CASH OUTFLOWS & & & & Gap is positive. & & \\
\hline Loans \& Advances & 6636.55 & Deposits & $6,584.86$ & & & & & \\
\hline Investments & 124.2 & Borrowings & 0.11 & & & & & \\
\hline $\begin{array}{l}\text { Foreign Currency } \\
\text { Assets }\end{array}$ & 56.78 & $\begin{array}{l}\text { Foreign Currency } \\
\text { Liabilities }\end{array}$ & 1.74 & & & & & \\
\hline Total & 6817.53 & Total & $6,586.71$ & & 230.82 & & & \\
\hline $\begin{array}{l}\text { Over } 1 \text { year and } \\
\text { upto } 3 \text { years CASH } \\
\text { INFLOWS }\end{array}$ & & CASH OUTFLOWS & & & & Gap is negative. & & \\
\hline Loans \& Advances & 3149 & Deposits & $11,214.99$ & & $-7,290.30$ & & & \\
\hline Investments & 625.14 & Borrowings & 65.45 & & & & & \\
\hline $\begin{array}{l}\text { Foreign Currency } \\
\text { Assets }\end{array}$ & 216 & $\begin{array}{l}\text { Foreign Currency } \\
\text { Liabilities }\end{array}$ & - & & & & & \\
\hline Total & 3990.14 & Total & $11,280.44$ & & & & & \\
\hline $\begin{array}{l}\text { Over } 3 \text { year and } \\
\text { upto } 5 \text { years } \\
\text { CASH INFLOWS }\end{array}$ & & CASH OUTFLOWS & & & & & & \\
\hline Loans \& Advances & 1347.16 & Deposits & 557.06 & & 2167.21 & Gap is positive. & & \\
\hline Investments & 1377.11 & Borrowings & - & & & & & \\
\hline $\begin{array}{l}\text { Foreign Currency } \\
\text { Assets }\end{array}$ & & $\begin{array}{l}\text { Foreign Currency } \\
\text { Liabilities }\end{array}$ & - & & & & & \\
\hline Total & 2724.27 & Total & 557.06 & & & & & \\
\hline $\begin{array}{l}\text { Over } 5 \text { years } \\
\text { CASH INFLOWS }\end{array}$ & & CASH OUTFLOWS & & & & & & \\
\hline Loans \& Advances & 2049.76 & Deposits & $2,137.86$ & & & Gap is positive. & & \\
\hline Investments & 4646.76 & Borrowings & 200 & & & & & \\
\hline $\begin{array}{l}\text { Foreign Currency } \\
\text { Assets }\end{array}$ & & $\begin{array}{l}\text { Foreign Currency } \\
\text { Liabilities }\end{array}$ & - & & & & & \\
\hline Total assets & 6696.52 & Total liabilities & 2337.86 & 4358.66 & & & & \\
\hline
\end{tabular}


In 2011, we can see that on day 1 there is a positive gap, so it satisfies RBI Norms. On 2-7 days i.e., on $2^{\text {nd }}$ time bucket it is negative it does not satisfy RBI Norms, this is similar in the case of next time bucket also. On the $3^{\text {rd }}$ time bucket there's a Negative gap if 33 percentage of total cash outflow. Here the bank has breached RBI tolerance limits. On the $4^{\text {th }}$ bucket onwards it is positive gap. After that there is a negative gap, for that bank should raise the fund from outside to reduce this negative gap. After that they have positive gap on next time buckets.

Maturity Pattern of key assets and liabilities:

As at 31 ${ }^{\text {st }}$ March, 2012:

\section{[in Crore]}

Exhibit 2:

\begin{tabular}{|c|c|c|c|c|c|c|c|c|c|c|c|}
\hline Particulars 2012 & Day 1 & 2-7 days & $\begin{array}{l}8-14 \\
\text { days }\end{array}$ & \multicolumn{2}{|c|}{ 15-28 days } & $\begin{array}{l}29 \text { days } \\
\text { and } \\
\text { upto } 3 \\
\text { months }\end{array}$ & $\begin{array}{l}\text { Over } 3 \\
\text { months } \\
\text { and upto } \\
6 \text { months }\end{array}$ & $\begin{array}{l}\text { Over } 6 \\
\text { months } \\
\text { and upto } \\
1 \text { year }\end{array}$ & $\begin{array}{l}\text { Over } 1 \\
\text { year and } \\
\text { upto } 3 \\
\text { years }\end{array}$ & $\begin{array}{l}\text { Over } 3 \\
\text { year and } \\
\text { upto } 5 \\
\text { years }\end{array}$ & $\begin{array}{l}\text { Over } 5 \\
\text { years }\end{array}$ \\
\hline \multicolumn{12}{|l|}{ Liabilities } \\
\hline Deposits & 198.34 & 1064.59 & 1370.76 & \multicolumn{2}{|l|}{1359.04} & 7805.7 & 4512.43 & 8237.65 & 10828.64 & 715.36 & 408.02 \\
\hline Borrowings & 88.79 & - & - & \multicolumn{2}{|l|}{-} & 50.93 & 18.36 & 18.42 & 138.45 & 73.24 & 200 \\
\hline Foreign Currency Liabilities & 176.96 & 8.79 & 6.33 & \multicolumn{2}{|l|}{11.29} & 109.81 & 91.16 & 132.38 & 64.92 & 45.33 & - \\
\hline Total & 464.09 & 1073.38 & 1377.09 & \multicolumn{2}{|c|}{1370.33} & 7966.44 & 4621.95 & 8388.45 & 11032.01 & 833.93 & 608.02 \\
\hline \multicolumn{12}{|l|}{ Assets } \\
\hline Loans \& Advances & 671.2 & 226.28 & 304.76 & \multicolumn{2}{|l|}{582.37} & 4538.62 & 4068.04 & 7373.45 & 3484.3 & 2555.51 & 3476.21 \\
\hline Investments & 1.37 & 271.59 & 158.06 & 64.67 & 378.47 & 113 & 315.11 & 993.32 & 1805.81 & 5298.47 & - \\
\hline Foreign Currency Assets & 251.21 & - & 48.06 & 17.08 & 118.93 & 93.02 & 20.48 & 108 & - & - & - \\
\hline Total & 923.78 & 497.87 & 510.88 & 664.12 & 5036.02 & 4274.06 & 7709.04 & 4585.62 & 4361.32 & 8774.68 & - \\
\hline
\end{tabular}

\begin{tabular}{|c|c|c|c|c|c|c|c|c|c|c|}
\hline Particulars & Day 1 & 2-7 days & $\begin{array}{l}\text { 8-14 } \\
\text { days }\end{array}$ & $15-28$ days & $\begin{array}{l}29 \text { days } \\
\text { and } \\
\text { upto } 3 \\
\text { months }\end{array}$ & $\begin{array}{l}\text { Over } 3 \\
\text { months } \\
\text { and upto } \\
6 \text { months }\end{array}$ & $\begin{array}{l}\text { Over } 6 \\
\text { months } \\
\text { and upto } \\
1 \text { year }\end{array}$ & $\begin{array}{l}\text { Over } 1 \\
\text { year and } \\
\text { upto } 3 \\
\text { years }\end{array}$ & $\begin{array}{l}\text { Over } 3 \\
\text { year and } \\
\text { upto } 5 \\
\text { years }\end{array}$ & $\begin{array}{l}\text { Over } 5 \\
\text { years }\end{array}$ \\
\hline GAP(Assets-Liabilities) & 459.69 & -575.51 & -866.21 & -706.21 & -2930.42 & -347.89 & -679.41 & -6446.39 & 3527.39 & 8166.66 \\
\hline
\end{tabular}

Source: Annual Report of SIB, March, 2012

\section{Maturity Ladder Based on Contractual Maturities 2012:}

\begin{tabular}{|l|l|l|l|l|l|l|l|}
\hline $\begin{array}{l}\text { DAY 1 } \\
\text { CASH INFLOWS }\end{array}$ & AMOUNT & CASH OUTFLOWS & AMOUNT & GAP & & $\begin{array}{l}\text { RBI TOLERANCE } \\
\text { LIMITS }\end{array}$ & REMARKS \\
\hline Loans and Advances & 671.2 & Deposits & 198.34 & & & $\begin{array}{l}\text { Negative Gap } \\
\text { should not be } \\
\text { more than } 5 \% \text { of } \\
\text { total outflows }\end{array}$ & $\begin{array}{l}\text { Gap is positive. RBI } \\
\text { Compliant }\end{array}$ \\
\hline Investments & 1.37 & Borrowings & 88.79 & & & & \\
\hline $\begin{array}{l}\text { Foreign Currency } \\
\text { Assets }\end{array}$ & 251.21 & $\begin{array}{l}\text { Foreign Currency } \\
\text { Liabilities }\end{array}$ & 176.96 & & & & \\
\hline Total & 923.78 & & & 464.09 & 459.69 & & \\
\hline
\end{tabular}




\begin{tabular}{|c|c|c|c|c|c|c|c|}
\hline $\begin{array}{l}\text { DAY 2-7 } \\
\text { CASH INFLOWS }\end{array}$ & & CASH OUTFLOWS & & & $\begin{array}{l}\text { Negative Gap should not } \\
\text { be more than } 10 \% \text { of total } \\
\text { outflows }\end{array}$ & & $\begin{array}{l}\text { Negative gap is } 53 \% \\
\text { of total outflow. So it } \\
\text { is not RBI Compliant }\end{array}$ \\
\hline Loans and Advances & 226.28 & Deposits & 1064.59 & & & & \\
\hline Investments & 271.59 & Borrowings & - & & & & \\
\hline $\begin{array}{l}\text { Foreign Currency } \\
\text { Assets }\end{array}$ & - & $\begin{array}{l}\text { Foreign Currency } \\
\text { Liabilities }\end{array}$ & 8.79 & & & & \\
\hline Total & 497.87 & & 1073.38 & -575.51 & & & \\
\hline $\begin{array}{l}\text { 8-14 days } \\
\text { CASH INFLOWS }\end{array}$ & & CASH OUTFLOWS & & & $\begin{array}{l}\text { Negative Gap should not } \\
\text { be more than } 15 \% \text { of total } \\
\text { outflows }\end{array}$ & & $\begin{array}{l}\text { Negative gap is } 62 \% \\
\text { of total cash outflow. } \\
\text { Here the Bank } \\
\text { has breached RBI } \\
\text { tolerance limits. }\end{array}$ \\
\hline Loans and Advances & 304.76 & Deposits & & 1370.76 & & & \\
\hline Loans and Advances & 304.76 & Deposits & & & & & \\
\hline Investments & 158.06 & Borrowings & & & & & \\
\hline $\begin{array}{l}\text { Foreign Currency } \\
\text { Assets }\end{array}$ & 48.06 & $\begin{array}{l}\text { Foreign Currency } \\
\text { Liabilities }\end{array}$ & & & & & \\
\hline Total & 510.88 & & -866.21 & & & & \\
\hline $\begin{array}{l}\text { 15-28days } \\
\text { CASH INFLOWS }\end{array}$ & & CASH OUTFLOWS & & & & & \\
\hline Loans \& Advances & 582.37 & Deposits & 1359.04 & & $\begin{array}{l}\text { Negative Gap should not } \\
\text { be more than } 20 \% \text { of the } \\
\text { cash outflows. }\end{array}$ & & \\
\hline Investments & 64.67 & Borrowings & - & & & & \\
\hline $\begin{array}{l}\text { Foreign Currency } \\
\text { Assets }\end{array}$ & 17.08 & $\begin{array}{l}\text { Foreign Currency } \\
\text { Liabilities }\end{array}$ & 11.29 & & & & \\
\hline Total & 664.12 & Total & $\begin{array}{l}1370.33 \\
\text { Gap: - } \\
706.21\end{array}$ & & & Gap is negative & \\
\hline $\begin{array}{l}29 \text { days and upto } 3 \\
\text { months } \\
\text { CASH INFLOWS }\end{array}$ & & CASH OUTFLOWS & & & & & \\
\hline Loans \& Advances & 4538.62 & Deposits & 7805.7 & & & & Gap is negative. \\
\hline Investments & 378.47 & Borrowings & 50.93 & & & & \\
\hline
\end{tabular}




\begin{tabular}{|c|c|c|c|c|c|c|}
\hline $\begin{array}{l}\text { Foreign Currency } \\
\text { Assets }\end{array}$ & 118.93 & $\begin{array}{l}\text { Foreign Currency } \\
\text { Liabilities }\end{array}$ & 109.81 & & & \\
\hline Total & 5036.02 & Total & 7966.44 & & -2930.42 & \\
\hline $\begin{array}{l}\text { Over } 3 \text { months and } \\
\text { upto } 6 \text { months } \\
\text { CASH INFLOWS }\end{array}$ & & CASH OUTFLOWS & & & & \\
\hline Loans \& Advances & 4068.04 & Deposits & 4512.43 & & & Gap is negative. \\
\hline Investments & 113 & Borrowings & 18.36 & & & \\
\hline $\begin{array}{l}\text { Foreign Currency } \\
\text { Assets }\end{array}$ & 93.02 & $\begin{array}{l}\text { Foreign Currency } \\
\text { Liabilities }\end{array}$ & 91.16 & & & \\
\hline Total & 4274.06 & Total & 4621.95 & & -347.89 & \\
\hline $\begin{array}{l}\text { Over } 6 \text { months and } \\
\text { upto } 1 \text { year } \\
\text { CASH INFLOWS }\end{array}$ & & CASH OUTFLOWS & & & & Gap is negative \\
\hline Loans \& Advances & 7373.45 & Deposits & 8237.65 & & & \\
\hline Investments & 315.11 & Borrowings & 18.42 & & & \\
\hline $\begin{array}{l}\text { Foreign Currency } \\
\text { Assets }\end{array}$ & 20.48 & $\begin{array}{l}\text { Foreign Currency } \\
\text { Liabilities }\end{array}$ & 132.38 & & & \\
\hline Total & 7709.04 & Total & 8388.45 & & -679.41 & \\
\hline $\begin{array}{l}\text { Over } 1 \text { year and } \\
\text { upto } 3 \text { years CASH } \\
\text { INFLOWS }\end{array}$ & & CASH OUTFLOWS & & & & Gap is negative. \\
\hline Loans \& Advances & 3484.3 & Deposits & 10828.64 & & & \\
\hline Investments & 993.32 & Borrowings & 138.45 & & & \\
\hline $\begin{array}{l}\text { Foreign Currency } \\
\text { Assets }\end{array}$ & 108 & $\begin{array}{l}\text { Foreign Currency } \\
\text { Liabilities }\end{array}$ & 64.92 & & & \\
\hline Total & 4585.62 & Total & 11032.01 & & -7447.59 & \\
\hline $\begin{array}{l}\text { Over } 3 \text { year and } \\
\text { upto } 5 \text { years } \\
\text { CASH INFLOWS }\end{array}$ & & CASH OUTFLOWS & & & & \\
\hline Loans \& Advances & 2555.51 & Deposits & 715.36 & & & Gap is positive. \\
\hline Investments & 1805.81 & Borrowings & 73.24 & & & \\
\hline $\begin{array}{l}\text { Foreign Currency } \\
\text { Assets }\end{array}$ & - & $\begin{array}{l}\text { Foreign Currency } \\
\text { Liabilities }\end{array}$ & 45.33 & & & \\
\hline Total & 4361.32 & Total & 833.93 & & -3527.39 & \\
\hline $\begin{array}{l}\text { Over } 5 \text { years } \\
\text { CASH INFLOWS }\end{array}$ & & CASH OUTFLOWS & & & & \\
\hline Loans \& Advances & 3476.21 & Deposits & 408.02 & & & Gap is positive. \\
\hline Investments & 5298.47 & Borrowings & 200 & & & \\
\hline $\begin{array}{l}\text { Foreign Currency } \\
\text { Assets }\end{array}$ & - & $\begin{array}{l}\text { Foreign Currency } \\
\text { Liabilities }\end{array}$ & - & & & \\
\hline Total & 8774.68 & Total & 608.02 & 8166.66 & & \\
\hline
\end{tabular}

In 2012 , on day 1 there is positive gap. After that the bank has negative gap i.e., on the $2^{\text {nd }}$ bucket onwards bank possess negative gap of $53 \%$ of total outflow. So it is not upto the RBI Compliant there is liquidity problem, bank won't be able to meet it's short term financial demands. From $9^{\text {th }}$ time bucket onwards there's a positive gap. 
Maturity Pattern of key assets and liabilities:

As at 31 ${ }^{\text {st }}$ March, 2013:

\section{[in Crore]}

\section{Exhibit 3}

\begin{tabular}{|c|c|c|c|c|c|c|c|c|c|c|}
\hline Particulars 2013 & Day 1 & $\begin{array}{l}2-7 \\
\text { days }\end{array}$ & $\begin{array}{l}\text { 8-14 } \\
\text { days }\end{array}$ & $\begin{array}{l}15-28 \\
\text { days }\end{array}$ & $\begin{array}{l}29 \text { days } \\
\text { and upto } 3 \\
\text { months }\end{array}$ & $\begin{array}{l}\text { Over } 3 \text { months } \\
\text { and upto } 6 \\
\text { months }\end{array}$ & $\begin{array}{l}\text { Over } 6 \\
\text { months and } \\
\text { upto } 1 \text { year }\end{array}$ & $\begin{array}{l}\text { Over } 1 \text { year } \\
\text { and upto } 3 \\
\text { years }\end{array}$ & $\begin{array}{l}\text { Over } 3 \text { year } \\
\text { and upto } 5 \\
\text { years }\end{array}$ & $\begin{array}{l}\text { Over } 5 \\
\text { years }\end{array}$ \\
\hline \multicolumn{11}{|l|}{ Liabilities } \\
\hline Deposits & 289.92 & 1114.09 & 1312.75 & 1281.37 & 8923.65 & 4620.22 & 9166.44 & 16104.02 & \begin{tabular}{|l|}
752.88 \\
\end{tabular} & 696.96 \\
\hline Borrowings & 220.83 & - & - & 65.06 & 81.49 & 296.4 & 128.03 & 164.67 & 128.07 & 200 \\
\hline $\begin{array}{l}\text { Foreign Currency } \\
\text { Liabilities }\end{array}$ & 257.3 & 1.78 & 3.12 & 6.33 & 111.48 & 321.88 & 186.54 & 86.15 & 48.59 & - \\
\hline Total & 768.05 & 1115.87 & 1315.87 & 1352.76 & 9116.62 & 5238.5 & 9481.01 & 16354.84 & 929.54 & 896.96 \\
\hline \multicolumn{11}{|l|}{ Assets } \\
\hline Loans \& Advances & 1009.88 & 240.66 & 389.47 & 785.76 & 4114.14 & 4449.64 & 9301.82 & 4580.83 & 3198 & 3745.33 \\
\hline Investments & 101.93 & 312.61 & 163.14 & 100.84 & 900.01 & 613.33 & 674.39 & 1894.05 & 2310.72 & 5452.45 \\
\hline $\begin{array}{l}\text { Foreign Currency } \\
\text { Assets }\end{array}$ & 309.9 & - & 14.57 & 21.4 & - & 138.01 & 23.35 & - & - & - \\
\hline Total & 1421.71 & 553.27 & 567.18 & 908 & 5014.15 & 5200.98 & 9999.56 & 6474.88 & 5508.72 & 9197.78 \\
\hline Particulars & Day 1 & \begin{tabular}{|l|}
$2-7$ \\
days
\end{tabular} & 8-14 days & $\begin{array}{l}15-28 \\
\text { days }\end{array}$ & $\begin{array}{l}29 \text { days } \\
\text { and upto } 3 \\
\text { months } \\
\end{array}$ & \begin{tabular}{|l} 
Over 3 months \\
and upto 6 \\
months \\
\end{tabular} & \begin{tabular}{|l|} 
Over 6 \\
months and \\
upto 1 year
\end{tabular} & $\begin{array}{l}\text { Over1 year } \\
\text { and upto } 3 \\
\text { years }\end{array}$ & \begin{tabular}{|l|} 
Over 3 year \\
and upto 5 \\
years
\end{tabular} & $\begin{array}{l}\text { Over } 5 \\
\text { years }\end{array}$ \\
\hline $\begin{array}{l}\text { GAP(Assets- } \\
\text { Liabilities) }\end{array}$ & 653.66 & -562.6 & -748.69 & -444.76 & -4102.47 & -37.52 & 518.55 & -9879.96 & 4579.18 & 8300.82 \\
\hline
\end{tabular}

Source: Annual Report of SIB: March, 2013

Maturity Ladder Based on Contractual Maturities 2013:

\begin{tabular}{|c|c|c|c|c|c|c|}
\hline $\begin{array}{l}\text { DAY } 1 \\
\text { CASH INFLOWS }\end{array}$ & AMOUNT & CASH OUTFLOWS & AMOUNT & GAP & RBI TOLERANCE LIMITS & REMARKS \\
\hline Loans and Advances & 1009.88 & Deposits & 289.92 & & \multirow{4}{*}{$\begin{array}{l}\text { Negative Gap should } \\
\text { not be more than } 5 \% \text { of } \\
\text { total outflows }\end{array}$} & \multirow{4}{*}{ Gap is positive. RBI compliant. } \\
\hline Investments & 101.93 & Borrowings & 220.83 & & & \\
\hline Foreign Currency Assets & 309.9 & $\begin{array}{l}\text { Foreign Currency } \\
\text { Liabilities }\end{array}$ & 257.3 & & & \\
\hline Total & 1421.71 & & 768.05 & 653.66 & & \\
\hline $\begin{array}{l}\text { DAY 2-7 } \\
\text { CASH INFLOWS }\end{array}$ & & CASH OUTFLOWS & & & \multirow{2}{*}{$\begin{array}{l}\text { Negative Gap should not } \\
\text { be more than } 10 \% \text { of } \\
\text { total outflows }\end{array}$} & \multirow{2}{*}{$\begin{array}{l}\text { Negative Gap is } 50 \% \text { of the total cash } \\
\text { outflows. So it is not RBI Compliant. }\end{array}$} \\
\hline Loans and Advances & 240.66 & Deposits & 1114.09 & & & \\
\hline Investments & 312.61 & Borrowings & - & Compliant. & & \\
\hline Foreign Currency Assets & - & $\begin{array}{l}\text { Foreign Currency } \\
\text { Liabilities }\end{array}$ & 1.78 & & & \\
\hline Total & 553.27 & & 1115.87 & -562.6 & & \\
\hline $\begin{array}{l}\text { 8-14 days } \\
\text { CASH INFLOWS }\end{array}$ & & CASH OUTFLOWS & & & \multirow{4}{*}{$\begin{array}{l}\text { Negative Gap should not } \\
\text { be more than } 15 \% \text { of } \\
\text { total outflows }\end{array}$} & \multirow{4}{*}{$\begin{array}{l}\text { Negative gap is } 56 \% \text { of the total cash } \\
\text { outflows. Here the Bank has breached } \\
\text { RBI tolerance limits. }\end{array}$} \\
\hline Loans and Advances & 389.47 & Deposits & 1312.75 & & & \\
\hline Investments & 163.14 & Borrowings & - & & & \\
\hline Foreign Currency Assets & 14.57 & $\begin{array}{l}\text { Foreign Currency } \\
\text { Liabilities }\end{array}$ & 3.12 & & & \\
\hline Total & 567.18 & & 1315.87 & -748.69 & & \\
\hline
\end{tabular}




\begin{tabular}{|c|c|c|c|c|c|c|}
\hline $\begin{array}{l}\text { 15-28days } \\
\text { CASH INFLOWS }\end{array}$ & & CASH OUTFLOWS & & & & \\
\hline Loans \& Advances & 785.76 & Deposits & 1281.37 & & \multirow{3}{*}{$\begin{array}{l}\text { Negative Gap should not } \\
\text { be more than } 20 \% \text { of } \\
\text { the cash outflows. }\end{array}$} & \multirow{3}{*}{$\begin{array}{l}\text { Negative gap is } 32 \% \text { of the total cash } \\
\text { outflows. So it is not RBI Compliant. }\end{array}$} \\
\hline Investments & 100.84 & Borrowings & 65.06 & & & \\
\hline Foreign Currency Assets & 21.4 & $\begin{array}{l}\text { Foreign Currency } \\
\text { Liabilities }\end{array}$ & 6.33 & & & \\
\hline Total & 908 & Total & 1352.76 & -444.76 & & \\
\hline $\begin{array}{l}29 \text { days and upto } 3 \text { months } \\
\text { CASH INFLOWS }\end{array}$ & & CASH OUTFLOWS & & & & \\
\hline Loans \& Advances & 4114.14 & Deposits & 8923.65 & & & Gap is negative. \\
\hline Investments & 900.01 & Borrowings & 81.49 & & & \\
\hline Foreign Currency Assets & - & $\begin{array}{l}\text { Foreign Currency } \\
\text { Liabilities }\end{array}$ & 111.48 & & & \\
\hline Total & 5014.15 & Total & 9116.62 & -4102.47 & & \\
\hline $\begin{array}{l}\text { Over } 3 \text { months and upto } 6 \\
\text { months } \\
\text { CASH INFLOWS }\end{array}$ & & CASH OUTFLOWS & & & & \\
\hline Loans \& Advances & 4449.64 & Deposits & 4620.22 & & Gap is negative. & \\
\hline Investments & 613.33 & Borrowings & 296.4 & & & \\
\hline Foreign Currency Assets & 138.01 & $\begin{array}{l}\text { Foreign Currency } \\
\text { Liabilities }\end{array}$ & 321.88 & & & \\
\hline Total & 5200.98 & Total & 5238.5 & -27.52 & & \\
\hline $\begin{array}{l}\text { Over } 6 \text { months and upto } \\
1 \text { year } \\
\text { CASH INFLOWS }\end{array}$ & & CASH OUTFLOWS & & & Gap is positive. & \\
\hline Loans \& Advances & 9301.82 & Deposits & 9166.44 & & & \\
\hline Investments & 674.39 & Borrowings & 128.03 & & & \\
\hline Foreign Currency Assets & 23.35 & $\begin{array}{l}\text { Foreign Currency } \\
\text { Liabilities }\end{array}$ & 186.54 & & & \\
\hline Total & 9999.56 & Total & 9481.01 & & & \\
\hline $\begin{array}{l}\text { Over } 1 \text { year and upto } 3 \\
\text { years CASH INFLOWS }\end{array}$ & & CASH OUTFLOWS & & & & \\
\hline Loans \& Advances & 4580.83 & Deposits & 16104.02 & & \multirow[t]{3}{*}{ Gap is negative. } & \\
\hline Investments & 1894.05 & Borrowings & 164.67 & & & \\
\hline Foreign Currency Assets & nil & $\begin{array}{l}\text { Foreign Currency } \\
\text { Liabilities }\end{array}$ & 86.15 & & & \\
\hline Total & 6474.88 & & 16354.84 & -9879.96 & & \\
\hline $\begin{array}{l}\text { Over } 3 \text { year and upto } 5 \\
\text { years } \\
\text { CASH INFLOWS }\end{array}$ & & CASH OUTFLOWS & & & & \\
\hline Loans \& Advances & 3198 & Deposits & 752.88 & & Gap is positive. & \\
\hline Investments & 2310.72 & Borrowings & 128.07 & & & \\
\hline Foreign Currency Assets & - & $\begin{array}{l}\text { Foreign Currency } \\
\text { Liabilities }\end{array}$ & 48.59 & & & \\
\hline Total & 5508.72 & Total & 929.54 & 4579.18 & & \\
\hline $\begin{array}{l}\text { Over } 5 \text { years } \\
\text { CASH INFLOWS }\end{array}$ & & CASH OUTFLOWS & & & & \\
\hline Loans \& Advances & 3745.33 & Deposits & 696.96 & & & \\
\hline Investments & 5452.45 & Borrowings & 200 & & Gap is positive. & \\
\hline Foreign Currency Assets & - & $\begin{array}{l}\text { Foreign Currency } \\
\text { Liabilities }\end{array}$ & - & & & \\
\hline Total & 9197.78 & Total & 896.96 & 8300.82 & & \\
\hline
\end{tabular}


In 2013 , on $2^{\text {nd }}$ time bucket, negative gap is 50 percentage of the total cash outflows. So it is not upto the RBI Compliant and here bank faces negative gap, and as a result to overcome that they have to raise funds from outside to meet their liabilities. On the $7^{\text {th }}$ time bucket they have a positive gap, after that there is a negative gap. After this level they maintained positive gap.

Maturity Pattern of key assets and liabilities:

As at 31 ${ }^{\text {st }}$ March, 2014:

[in Crore]

\section{Exhibit 4}

\begin{tabular}{|c|c|c|c|c|c|c|c|c|c|c|}
\hline $\begin{array}{l}\text { Particulars } \\
2014\end{array}$ & Day 1 & 2-7 days & $\begin{array}{l}8-14 \\
\text { days }\end{array}$ & $\begin{array}{l}15-28 \\
\text { days }\end{array}$ & $\begin{array}{l}29 \text { days } \\
\text { and upto } 3 \\
\text { months }\end{array}$ & $\begin{array}{l}\text { Over } 3 \text { months } \\
\text { and upto } 6 \\
\text { months }\end{array}$ & $\begin{array}{l}\text { Over } 6 \\
\text { months and } \\
\text { upto } 1 \text { year }\end{array}$ & $\begin{array}{l}\text { Over } 1 \text { year } \\
\text { and upto } 3 \\
\text { years }\end{array}$ & $\begin{array}{l}\text { Over } 3 \text { years } \\
\text { and upto } 5 \\
\text { years }\end{array}$ & $\begin{array}{l}\text { Over } 5 \\
\text { years }\end{array}$ \\
\hline \multicolumn{11}{|l|}{ Liabilities } \\
\hline Deposits & 212.5 & 633.25 & 539.44 & 2015.84 & 13336.21 & 5106.59 & 6209.33 & 12324.83 & 1302.02 & 5811.08 \\
\hline Borrowings & 19.85 & 84.91 & - & 0.48 & 0.06 & 791.54 & 514.23 & 877.87 & 241.84 & 200 \\
\hline $\begin{array}{l}\text { Foreign } \\
\text { Currency } \\
\text { Liabilities }\end{array}$ & 10.94 & 3.72 & 4.04 & 7.4 & 40.12 & 456.55 & 513.26 & 115.58 & 326.19 & - \\
\hline Total & 243.29 & 721.88 & 543.48 & 2023.72 & 13376.39 & 6354.68 & 7236.82 & 13318.28 & 1870.05 & 6011.08 \\
\hline \multicolumn{11}{|l|}{ Assets } \\
\hline $\begin{array}{l}\text { Loans \& } \\
\text { Advances }\end{array}$ & 1161.95 & 335.07 & 388.94 & 1006.56 & 4998.97 & 4393.23 & 10631.96 & 5276.98 & 3258.12 & 4778.08 \\
\hline Investments & 99.03 & -42.31 & 70.8 & 108.7 & 786 & 262.77 & 745.29 & 2712.8 & 2217.41 & 7391.29 \\
\hline $\begin{array}{l}\text { Foreign } \\
\text { Currency Assets }\end{array}$ & 41.24 & 41.94 & 0.72 & 22.98 & 69.93 & 154.12 & 329.56 & - & 0.71 & - \\
\hline Total & 1302.22 & 334.7 & 460.46 & 1138.24 & 5854.9 & 4810.12 & 11706.81 & 7989.78 & 5476.24 & 12169.37 \\
\hline
\end{tabular}

\begin{tabular}{|c|c|c|c|c|c|c|c|c|c|c|}
\hline Particulars & Day 1 & 2-7 days & $\begin{array}{l}8-14 \\
\text { days }\end{array}$ & $\begin{array}{l}15-28 \\
\text { days }\end{array}$ & $\begin{array}{l}29 \text { days } \\
\text { and upto } 3 \\
\text { months }\end{array}$ & $\begin{array}{l}\text { Over } 3 \text { months } \\
\text { and upto } 6 \\
\text { months }\end{array}$ & $\begin{array}{l}\text { Over } 6 \\
\text { months and } \\
\text { upto } 1 \text { year }\end{array}$ & $\begin{array}{l}\text { Over } 1 \text { year } \\
\text { and upto } 3 \\
\text { years }\end{array}$ & $\begin{array}{l}\text { Over } 3 \text { year } \\
\text { and upto } 5 \\
\text { years }\end{array}$ & $\begin{array}{l}\text { Over } 5 \\
\text { years }\end{array}$ \\
\hline $\begin{array}{l}\text { GAP(Assets- } \\
\text { Liabilities) }\end{array}$ & 1058.93 & -387.18 & -83.02 & -885.48 & -7521.49 & -1544.56 & 4469.99 & -5328.5 & 3606.19 & 6158.29 \\
\hline
\end{tabular}

Source: Annual Report, SIB, March, 2014

Maturity Ladder Based on Contractual Maturities 2014:

\begin{tabular}{|c|c|c|c|c|c|c|}
\hline $\begin{array}{l}\text { DAY } 1 \\
\text { CASH INFLOWS }\end{array}$ & AMOUNT & CASH OUTFLOWS & AMOUNT & GAP & RBI TOLERANCE LIMITS & REMARKS \\
\hline Loans and Advances & 1161.95 & Deposits & 212.5 & & \multirow{4}{*}{$\begin{array}{l}\text { Negative Gap should not be } \\
\text { more than } 5 \% \text { of total outflows }\end{array}$} & \multirow{4}{*}{ Gap is positive. RBI Compliant. } \\
\hline Investments & 99.03 & Borrowings & 19.85 & & & \\
\hline $\begin{array}{l}\text { Foreign Currency } \\
\text { Assets }\end{array}$ & 41.24 & $\begin{array}{l}\text { Foreign Currency } \\
\text { Liabilities }\end{array}$ & 10.94 & & & \\
\hline Total & 1302.22 & & 243.29 & 1058.93 & & \\
\hline $\begin{array}{l}\text { DAY 2-7 } \\
\text { CASH INFLOWS }\end{array}$ & & CASH OUTFLOWS & & & \multirow{5}{*}{$\begin{array}{l}\text { Negative Gap should not be } \\
\text { more than } 10 \% \text { of total outflows }\end{array}$} & \multirow{5}{*}{$\begin{array}{l}\text { Negative gap is } 53 \% \text { of the total cash } \\
\text { outflows. So it is not RBI Compliant. }\end{array}$} \\
\hline Loans and Advances & 335.07 & Deposits & 633.25 & & & \\
\hline Investments & -42.31 & Borrowings & 84.91 & & & \\
\hline $\begin{array}{l}\text { Foreign Currency } \\
\text { Assets }\end{array}$ & 41.94 & $\begin{array}{l}\text { Foreign Currency } \\
\text { Liabilities }\end{array}$ & 3.72 & & & \\
\hline Total & 334.7 & & 721.88 & -387.18 & & \\
\hline
\end{tabular}




\begin{tabular}{|c|c|c|c|c|c|c|}
\hline $\begin{array}{l}\text { 8-14 days } \\
\text { CASH INFLOWS }\end{array}$ & & CASH OUTFLOWS & & & \multirow{5}{*}{$\begin{array}{l}\text { Negative Gap should not be } \\
\text { more than } 15 \% \text { of total outflows }\end{array}$} & \multirow{5}{*}{$\begin{array}{l}\text { Negative gap is } 16 \% \text { of the total } \\
\text { cash outflows. Here the Bank has } \\
\text { breached RBI tolerance limits. }\end{array}$} \\
\hline Loans and Advances & 388.94 & Deposits & 539.44 & & & \\
\hline Investments & 70.8 & Borrowings & - & & & \\
\hline $\begin{array}{l}\text { Foreign Currency } \\
\text { Assets }\end{array}$ & 0.72 & $\begin{array}{l}\text { Foreign Currency } \\
\text { Liabilities }\end{array}$ & 4.04 & & & \\
\hline Total & 460.46 & & 543.48 & -83.02 & & \\
\hline $\begin{array}{l}\text { 15-28days } \\
\text { CASH INFLOWS }\end{array}$ & & CASH OUTFLOWS & & & \multirow{5}{*}{$\begin{array}{l}\text { Negative gap should not be } \\
\text { more than } 20 \% \text { of the total cash } \\
\text { outflows. }\end{array}$} & \multirow{5}{*}{$\begin{array}{l}\text { Negative gap is } 43 \% \text { of the total cash } \\
\text { outflows. So it is not RBI Compliant }\end{array}$} \\
\hline Loans \& Advances & 1006.56 & Deposits & 2015.84 & & & \\
\hline Investments & 108.7 & Borrowings & 0.48 & & & \\
\hline $\begin{array}{l}\text { Foreign Currency } \\
\text { Assets }\end{array}$ & 22.98 & $\begin{array}{l}\text { Foreign Currency } \\
\text { Liabilities }\end{array}$ & 7.4 & & & \\
\hline Total & 1138.24 & Total & 2023.72 & -885.48 & & \\
\hline $\begin{array}{l}29 \text { days and upto } 3 \\
\text { months } \\
\text { CASH INFLOWS }\end{array}$ & & CASH OUTFLOWS & & & & \multirow{5}{*}{ Gap is negative. } \\
\hline Loans \& Advances & 4998.97 & Deposits & 13336.21 & & & \\
\hline Investments & 786 & Borrowings & 0.06 & & & \\
\hline $\begin{array}{l}\text { Foreign Currency } \\
\text { Assets }\end{array}$ & 69.93 & $\begin{array}{l}\text { Foreign Currency } \\
\text { Liabilities }\end{array}$ & 40.12 & & & \\
\hline Total & 5854.9 & Total & 13376.39 & -7521.49 & & \\
\hline $\begin{array}{l}\text { Over } 3 \text { months and } \\
\text { upto } 6 \text { months } \\
\text { CASH INFLOWS }\end{array}$ & & CASH OUTFLOWS & & & & \multirow{5}{*}{ Gap is negative. } \\
\hline Loans \& Advances & 4393.23 & Deposits & 5106.59 & & & \\
\hline Investments & 262.77 & Borrowings & 791.54 & & & \\
\hline $\begin{array}{l}\text { Foreign Currency } \\
\text { Assets }\end{array}$ & 154.12 & $\begin{array}{l}\text { Foreign Currency } \\
\text { Liabilities }\end{array}$ & 456.55 & & & \\
\hline Total & 4810.12 & Total & 6354.68 & -1544.56 & & \\
\hline $\begin{array}{l}\text { Over } 6 \text { months and } \\
\text { upto } 1 \text { year } \\
\text { CASH INFLOWS }\end{array}$ & & CASH OUTFLOWS & & & & \multirow{5}{*}{ Gap is positive. } \\
\hline Loans \& Advances & 10631.96 & Deposits & 6209.33 & & & \\
\hline Investments & 745.29 & Borrowings & 514.23 & & & \\
\hline $\begin{array}{l}\text { Foreign Currency } \\
\text { Assets }\end{array}$ & 329.56 & $\begin{array}{l}\text { Foreign Currency } \\
\text { Liabilities }\end{array}$ & 513.26 & & & \\
\hline Total & 11706.81 & Total & 7236.82 & 4469.99 & & \\
\hline $\begin{array}{l}\text { Over } 1 \text { year and } \\
\text { upto } 3 \text { years CASH } \\
\text { INFLOWS }\end{array}$ & & CASH OUTFLOWS & & & & \multirow{5}{*}{ Gap is negative. } \\
\hline Loans \& Advances & 5276.98 & Deposits & 12324.83 & & & \\
\hline Investments & 2712.8 & Borrowings & 877.87 & & & \\
\hline $\begin{array}{l}\text { Foreign Currency } \\
\text { Assets }\end{array}$ & - & $\begin{array}{l}\text { Foreign Currency } \\
\text { Liabilities }\end{array}$ & 115.58 & & & \\
\hline Total & 7989.78 & Total & 13318.28 & -5328.5 & & \\
\hline $\begin{array}{l}\text { Over } 3 \text { year and } \\
\text { upto } 5 \text { years } \\
\text { CASH INFLOWS }\end{array}$ & & CASH OUTFLOWS & & & & \multirow{3}{*}{ Gap is positive. } \\
\hline Loans \& Advances & 3258.12 & Deposits & 1302.02 & & & \\
\hline Investments & 2217.41 & Borrowings & 241.84 & & & \\
\hline
\end{tabular}




\begin{tabular}{|c|c|c|c|c|c|c|}
\hline $\begin{array}{l}\text { Foreign Currency } \\
\text { Assets }\end{array}$ & 0.71 & $\begin{array}{l}\text { Foreign Currency } \\
\text { Liabilities }\end{array}$ & 326.19 & & & \\
\hline Total & 5476.24 & Total & 1870.05 & 3606.19 & & \\
\hline $\begin{array}{l}\text { Over } 5 \text { years } \\
\text { CASH INFLOWS }\end{array}$ & & CASH OUTFLOWS & & & & \multirow{9}{*}{$\begin{array}{l}\text { Negative gap is } 43 \% \text { of the total cash } \\
\text { outflows. So it is not in tolerance } \\
\text { limit. }\end{array}$} \\
\hline Loans \& Advances & 4778.08 & Deposits & 5811.08 & & & \\
\hline Investments & 7391.29 & Borrowings & 200 & & & \\
\hline $\begin{array}{l}\text { Foreign Currency } \\
\text { Assets }\end{array}$ & - & $\begin{array}{l}\text { Foreign Currency } \\
\text { Liabilities }\end{array}$ & - & & & \\
\hline Total & 12169.37 & Total & 6011.08 & 6158.29 & & \\
\hline Loans \& Advances & 1006.56 & Deposits & 2015.84 & & \multirow{4}{*}{$\begin{array}{l}\text { Negative gap should not be } \\
\text { more than } 20 \% \text { of the total cash } \\
\text { outflows. }\end{array}$} & \\
\hline Investments & 108.7 & Borrowings & 0.48 & & & \\
\hline $\begin{array}{l}\text { Foreign Currency } \\
\text { Assets }\end{array}$ & 22.98 & $\begin{array}{l}\text { Foreign Currency } \\
\text { Liabilities }\end{array}$ & 7.4 & & & \\
\hline Total & 1138.24 & Total & 2023.72 & -885.48 & & \\
\hline $\begin{array}{l}29 \text { days and upto } 3 \\
\text { months } \\
\text { CASH INFLOWS }\end{array}$ & & CASH OUTFLOWS & & & & \multirow{5}{*}{ Gap is negative. } \\
\hline Loans \& Advances & 4998.97 & Deposits & 13336.21 & & & \\
\hline Investments & 786 & Borrowings & 0.06 & & & \\
\hline $\begin{array}{l}\text { Foreign Currency } \\
\text { Assets }\end{array}$ & 69.93 & $\begin{array}{l}\text { Foreign Currency } \\
\text { Liabilities }\end{array}$ & 40.12 & & & \\
\hline Total & 5854.9 & Total & 13376.39 & -7521.49 & & \\
\hline $\begin{array}{l}\text { Over } 3 \text { months and } \\
\text { upto } 6 \text { months } \\
\text { CASH INFLOWS }\end{array}$ & & CASH OUTFLOWS & & & & \multirow{5}{*}{ Gap is negative. } \\
\hline Loans \& Advances & 4393.23 & Deposits & 5106.59 & & & \\
\hline Investments & 262.77 & Borrowings & 791.54 & & & \\
\hline $\begin{array}{l}\text { Foreign Currency } \\
\text { Assets }\end{array}$ & 154.12 & $\begin{array}{l}\text { Foreign Currency } \\
\text { Liabilities }\end{array}$ & 456.55 & & & \\
\hline Total & 4810.12 & Total & 6354.68 & -1544.56 & & \\
\hline $\begin{array}{l}\text { Over } 6 \text { months and } \\
\text { upto } 1 \text { year } \\
\text { CASH INFLOWS }\end{array}$ & & CASH OUTFLOWS & & & & \multirow{6}{*}{ Gap is positive. } \\
\hline Loans \& Advances & 10631.96 & Deposits & 6209.33 & & & \\
\hline Investments & 745.29 & Borrowings & 514.23 & & & \\
\hline Investments & 745.29 & Borrowings & 514.23 & & & \\
\hline $\begin{array}{l}\text { Foreign Currency } \\
\text { Assets }\end{array}$ & 329.56 & $\begin{array}{l}\text { Foreign Currency } \\
\text { Liabilities }\end{array}$ & 513.26 & & & \\
\hline Total & 11706.81 & Total & 7236.82 & 4469.99 & & \\
\hline $\begin{array}{l}\text { Over } 1 \text { year and } \\
\text { upto } 3 \text { years CASH } \\
\text { INFLOWS }\end{array}$ & & CASH OUTFLOWS & & & & \multirow{5}{*}{ Gap is negative. } \\
\hline Loans \& Advances & 5276.98 & Deposits & 12324.83 & & & \\
\hline Investments & 2712.8 & Borrowings & 877.87 & & & \\
\hline $\begin{array}{l}\text { Foreign Currency } \\
\text { Assets }\end{array}$ & - & $\begin{array}{l}\text { Foreign Currency } \\
\text { Liabilities }\end{array}$ & 115.58 & & & \\
\hline Total & 7989.78 & Total & 13318.28 & -5328.5 & & \\
\hline
\end{tabular}




\begin{tabular}{|l|l|l|l|l|l|l|}
\hline $\begin{array}{l}\text { Over } 3 \text { year and } \\
\text { upto 5 years } \\
\text { CASH INFLOWS }\end{array}$ & CASH OUTFLOWS & & & \\
\hline Loans \& Advances & 3258.12 & Deposits & 1302.02 & & & Gap is positive. \\
\hline Investments & 2217.41 & Borrowings & 241.84 & & & \\
\hline $\begin{array}{l}\text { Foreign Currency } \\
\text { Assets }\end{array}$ & 0.71 & $\begin{array}{l}\text { Foreign Currency } \\
\text { Liabilities }\end{array}$ & 326.19 & & & \\
\hline Total & 5476.24 & Total & 1870.05 & 3606.19 & & Gap is positive. \\
\hline $\begin{array}{l}\text { Over 5 years } \\
\text { CASH INFLOWS }\end{array}$ & & CASH OUTFLOWS & & & & \\
\hline Loans \& Advances & 4778.08 & Deposits & 5811.08 & & & \\
\hline Investments & 7391.29 & Borrowings & 200 & & & \\
\hline $\begin{array}{l}\text { Foreign Currency } \\
\text { Assets }\end{array}$ & - & $\begin{array}{l}\text { Foreign Currency } \\
\text { Liabilities }\end{array}$ & - & & & \\
\hline Total & 12169.37 & Total & 6011.08 & 6158.29 & & \\
\hline
\end{tabular}

In 2014, on the $1^{\text {st }}$ time bucket they have a positive gap and they satisfy RBI Compliant. On $2^{\text {nd }}$ time bucket i.e., 2-7 day there is 53 percentage of negative gap this cannot satisfy RBI Compliant. After this they face liquidity problems in the further buckets. Then over 6 months and up to 1 year they have a positive gap. On the next time bucket they have a negative gap and they cover this gap on the succeeding time buckets.

Maturity Pattern of key assets and liabilities:

As at 31 ${ }^{\text {st }}$ March, 2015:

[in Crore]

Exhibit 5

\begin{tabular}{|c|c|c|c|c|c|c|c|c|c|c|}
\hline Particulars 2015 & Day 1 & $\begin{array}{l}2-7 \\
\text { days }\end{array}$ & $\begin{array}{l}8-14 \\
\text { days }\end{array}$ & $\begin{array}{l}15-28 \\
\text { days }\end{array}$ & $\begin{array}{l}29 \text { days } \\
\text { and upto } \\
3 \text { months }\end{array}$ & $\begin{array}{l}\text { Over } 3 \text { months } \\
\text { and upto } 6 \\
\text { months }\end{array}$ & $\begin{array}{l}\text { Over } 6 \\
\text { months and } \\
\text { upto } 1 \text { year }\end{array}$ & $\begin{array}{l}\text { Over } 1 \text { year } \\
\text { and upto } 3 \\
\text { years }\end{array}$ & $\begin{array}{l}\text { Over } 3 \text { year } \\
\text { and upto } 5 \\
\text { years }\end{array}$ & $\begin{array}{l}\text { Over } 5 \\
\text { years }\end{array}$ \\
\hline \multicolumn{11}{|l|}{ Liabilities } \\
\hline Deposits & 137.06 & 1120.97 & 842.63 & 2915.84 & 11270.62 & 10270.38 & 10497.58 & 6337.7 & 952.78 & 7566.93 \\
\hline Borrowings & 76.48 & - & 500 & - & 150 & 94.47 & 781.97 & 341.25 & 88.3 & 200 \\
\hline $\begin{array}{l}\text { Foreign Currency } \\
\text { Liabilities }\end{array}$ & 20.84 & 1.62 & 1.64 & 4.71 & 194.9 & 102.12 & 809.5 & 167.65 & 418.26 & - \\
\hline Total & 234.38 & 1122.59 & 1344.27 & 2920.55 & 11615.52 & 10466.97 & 12089.05 & 6846.6 & 1459.34 & 7766.93 \\
\hline \multicolumn{11}{|l|}{ Assets } \\
\hline Loans \& Advances & 2067.01 & 233.53 & 323.95 & 814.37 & 4379.4 & 4042.54 & 8985.38 & 5993.77 & 4166.06 & 6385.63 \\
\hline Investments & 288.96 & 1422.25 & 234.91 & 52.98 & 959.91 & 576.75 & 697.22 & 3619.44 & 1931.79 & 6932.96 \\
\hline $\begin{array}{l}\text { Foreign Currency } \\
\text { Assets }\end{array}$ & 107.23 & - & 1.25 & 5.8 & 82.45 & 281.81 & 428.87 & 4.23 & 0.01 & - \\
\hline Total & 2463.2 & 1655.78 & 560.11 & 873.15 & 5421.76 & 4901.1 & 10111.47 & 9617.44 & 6097.86 & 13318.59 \\
\hline Particulars & Day 1 & $\begin{array}{l}2-7 \\
\text { days }\end{array}$ & $\begin{array}{l}8-14 \\
\text { days }\end{array}$ & $\begin{array}{l}15-28 \\
\text { days }\end{array}$ & $\begin{array}{l}29 \text { days } \\
\text { and upto } \\
3 \text { months }\end{array}$ & $\begin{array}{l}\text { Over } 3 \text { months } \\
\text { and upto } 6 \\
\text { months }\end{array}$ & $\begin{array}{l}\text { Over } 6 \\
\text { months and } \\
\text { upto } 1 \text { year }\end{array}$ & $\begin{array}{l}\text { Over } 1 \text { year } \\
\text { and upto } 3 \\
\text { years }\end{array}$ & $\begin{array}{l}\text { Over } 3 \text { year } \\
\text { and upto } 5 \\
\text { years }\end{array}$ & $\begin{array}{l}\text { Over } 5 \\
\text { years }\end{array}$ \\
\hline $\begin{array}{l}\text { GAP(Assets- } \\
\text { Liabilities }\end{array}$ & 2228.82 & 533.19 & -784.16 & -2047.4 & -6193.76 & -5565.87 & -1977.58 & 2770.84 & 4638.52 & 5551.66 \\
\hline
\end{tabular}

Source: Annual Report, SIB, March, 2015 
Maturity Ladder Based on Contractual Maturities 2015:

\begin{tabular}{|c|c|c|c|c|c|c|}
\hline CASH OUTFLOWS & AMOUNT & & AMOUNT & & RBI TOLERANCE LIMITS & REMARKS \\
\hline Loans and Advances & 2067.01 & Deposits & 137.06 & & \multirow{4}{*}{$\begin{array}{l}\text { Negative Gap should not be more } \\
\text { than } 5 \% \text { of total outflows }\end{array}$} & \multirow{4}{*}{ Gap is positive. RBI Compliant. } \\
\hline Investments & 288.96 & Borrowings & 76.48 & & & \\
\hline Foreign Currency Assets & 107.23 & $\begin{array}{l}\text { Foreign Currency } \\
\text { Liabilities }\end{array}$ & 20.84 & & & \\
\hline Total & 2463.2 & & 234.38 & 2228.82 & & \\
\hline $\begin{array}{l}\text { DAY 2-7 } \\
\text { CASH INFLOWS }\end{array}$ & & $\begin{array}{l}\text { CASH } \\
\text { OUTFLOWS }\end{array}$ & & & \multirow{5}{*}{$\begin{array}{l}\text { Negative Gap should not be more } \\
\text { than } 10 \% \text { of total outflows } \\
\text { Gap is positive. RBI Compliant. }\end{array}$} & \\
\hline Loans and Advances & 233.53 & Deposits & 1120.97 & & & \\
\hline Investments & 1422.25 & Borrowings & - & & & \\
\hline Foreign Currency Assets & - & $\begin{array}{l}\text { Foreign Currency } \\
\text { Liabilities }\end{array}$ & 1.62 & & & \\
\hline Total & 1655.78 & & 1122.59 & 533.19 & & \\
\hline $\begin{array}{l}\text { 8-14 days } \\
\text { CASH INFLOWS }\end{array}$ & & $\begin{array}{l}\text { CASH } \\
\text { OUTFLOWS }\end{array}$ & & & \multirow{5}{*}{$\begin{array}{l}\text { Negative Gap should not be more } \\
\text { than } 15 \% \text { of total outflows }\end{array}$} & \multirow{5}{*}{$\begin{array}{l}\text { Negative gap is } 58 \% \text { of the total } \\
\text { cash outflows. So it is not RBI } \\
\text { Compliant. }\end{array}$} \\
\hline Loans and Advances & 323.95 & Deposits & 842.63 & & & \\
\hline Investments & 234.91 & Borrowings & 500 & & & \\
\hline Foreign Currency Assets & 1.25 & $\begin{array}{l}\text { Foreign Currency } \\
\text { Liabilities }\end{array}$ & 1.64 & & & \\
\hline Total & 560.11 & & 1344.27 & -784.16 & & \\
\hline $\begin{array}{l}\text { 15-28days } \\
\text { CASH INFLOWS }\end{array}$ & & $\begin{array}{l}\text { CASH } \\
\text { OUTFLOWS }\end{array}$ & & & & \\
\hline Loans \& Advances & 814.37 & Deposits & 2915.84 & & \multirow{4}{*}{$\begin{array}{l}\text { Negative Gap should not be more } \\
\text { than } 20 \% \text { of the cash outflows }\end{array}$} & \multirow{4}{*}{$\begin{array}{l}\text { Negative gap is } 70 \% \text { of the total } \\
\text { cash outflows. So it is not RBI } \\
\text { Compliant. }\end{array}$} \\
\hline Investments & 52.98 & Borrowings & - & & & \\
\hline Foreign Currency Assets & 5.8 & $\begin{array}{l}\text { Foreign Currency } \\
\text { Liabilities }\end{array}$ & 4.71 & & & \\
\hline Total & 873.15 & Total & 2920.55 & -2047.40 & & \\
\hline $\begin{array}{l}29 \text { days and upto } 3 \\
\text { months } \\
\text { CASH INFLOWS }\end{array}$ & & $\begin{array}{l}\text { CASH } \\
\text { OUTFLOWS }\end{array}$ & & & & \\
\hline Loans \& Advances & 4379.4 & Deposits & 11270.62 & & \multirow{5}{*}{ Gap is negative. } & \\
\hline Investments & 959.91 & Borrowings & 150 & & & \\
\hline Investments & 959.91 & Borrowings & 150 & & & \\
\hline Foreign Currency Assets & 82.45 & $\begin{array}{l}\text { Foreign Currency } \\
\text { Liabilities }\end{array}$ & 194.9 & & & \\
\hline Total & 5421.76 & Total & 11615.52 & -6193.76 & & \\
\hline $\begin{array}{l}\text { Over } 3 \text { months and upto } \\
6 \text { months } \\
\text { CASH INFLOWS }\end{array}$ & & $\begin{array}{l}\text { CASH } \\
\text { OUTFLOWS }\end{array}$ & & & \multirow{5}{*}{ Gap is negative. } & \\
\hline Loans \& Advances & 4042.54 & Deposits & 10270.38 & & & \\
\hline Investments & 576.75 & Borrowings & 94.47 & & & \\
\hline Foreign Currency Assets & 281.81 & $\begin{array}{l}\text { Foreign Currency } \\
\text { Liabilities }\end{array}$ & 102.12 & & & \\
\hline Total & 4901.1 & Total & 10466.97 & -5565.87 & & \\
\hline
\end{tabular}




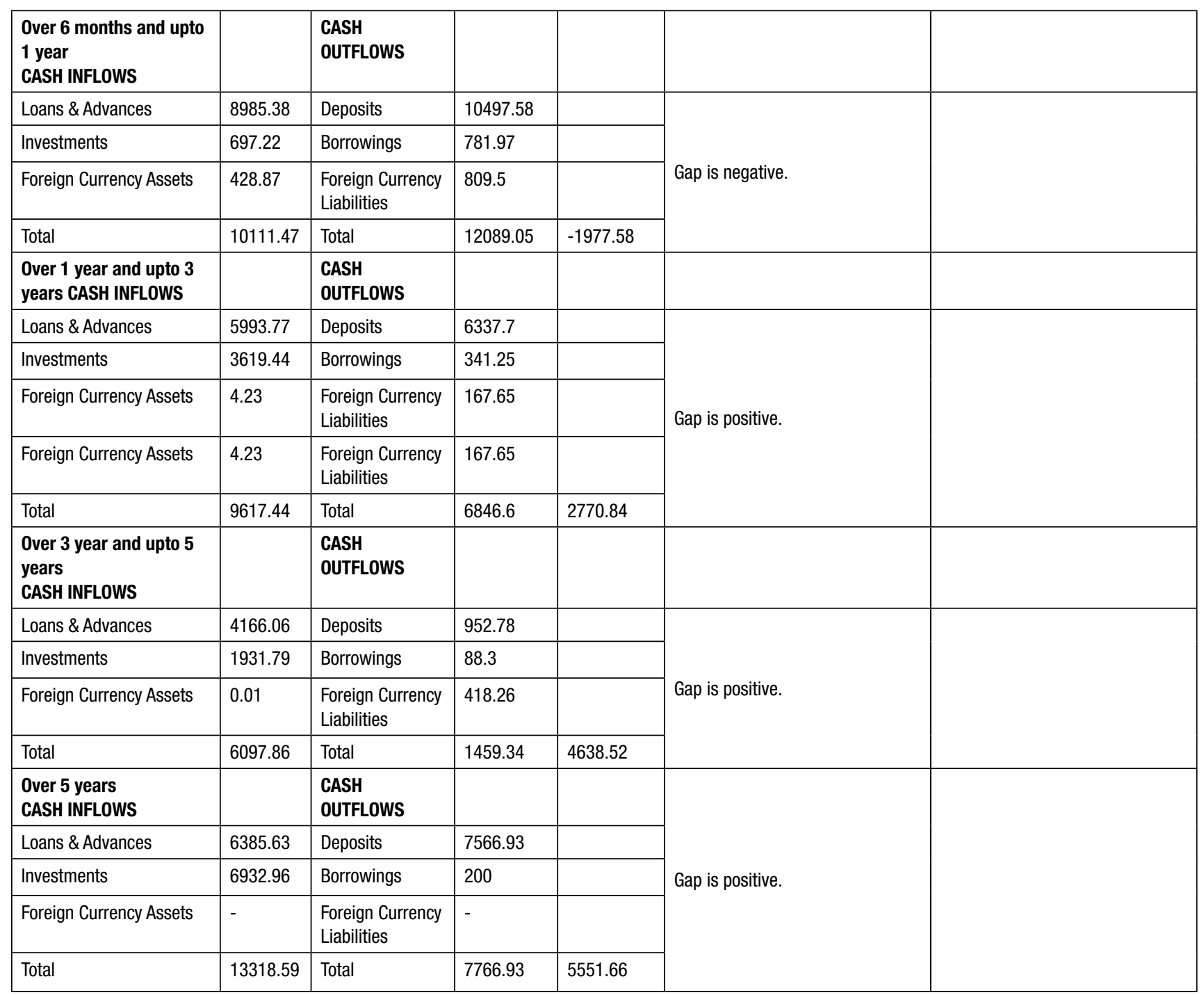

In 2015 , on the $1^{\text {st }}$ and $2^{\text {nd }}$ time bucket there is positive gap and Bank could satisfy RBI Compliance. On the $3^{\text {rd }}$ time bucket onwards there's a negative gap of 70 percentage of the total cash outflows. So, it is not upto the RBI Compliant, this arises due to holding a valuable asset that cannot be tradable when they need cash during to the lack of buyers. On the $7^{\text {th }}$ time bucket onwards, the bank keeps enough liquidity.

Maturity Pattern of key assets and liabilities:

As at 31st March, 2016:

[in Crore]

Exhibit 6

\begin{tabular}{|l|l|l|l|l|l|l|l|l|l|l|}
\hline $\begin{array}{l}\text { Particulars } \\
\text { 2016 }\end{array}$ & Day 1 & $\mathbf{2 - 7}$ days & $\begin{array}{l}\mathbf{8 - 1 4} \\
\text { days }\end{array}$ & $\begin{array}{l}\mathbf{1 5 - 2 8} \\
\text { days }\end{array}$ & $\begin{array}{l}\text { 29 days } \\
\text { and upto 3 } \\
\text { months }\end{array}$ & $\begin{array}{l}\text { Over 3 months } \\
\text { and upto 6 } \\
\text { months }\end{array}$ & $\begin{array}{l}\text { Over 6 } \\
\text { months and } \\
\text { upto 1 year }\end{array}$ & $\begin{array}{l}\text { Over 1 year } \\
\text { and upto 3 } \\
\text { years }\end{array}$ & $\begin{array}{l}\text { Over 3 year } \\
\text { and upto 5 } \\
\text { years }\end{array}$ & $\begin{array}{l}\text { Over 5 } \\
\text { years }\end{array}$ \\
\hline Liabilities & & & & & & & & & & \\
\hline Deposits & 212.74 & 930.69 & 863.88 & 1290.92 & 8709.88 & 6860.07 & 16672.03 & 6152.9 & 1229.61 & 12798.01 \\
\hline Borrowings & 63.69 & - & - & - & 927.57 & 336.27 & 170.63 & 316.79 & 200 & 300 \\
\hline
\end{tabular}




\begin{tabular}{|c|c|c|c|c|c|c|c|c|c|c|}
\hline $\begin{array}{l}\text { Foreign } \\
\text { Currency } \\
\text { Liabilities }\end{array}$ & 36.84 & 3.48 & 2.93 & 12.53 & 990 & 307.53 & 165.14 & 391.93 & 403.52 & - \\
\hline Total & 313.27 & 934.17 & 866.81 & 1303.45 & 10627.45 & 7503.87 & 17007.8 & 6861.62 & 1833.13 & 13098.01 \\
\hline \multicolumn{11}{|l|}{ Assets } \\
\hline $\begin{array}{l}\text { Loans \& } \\
\text { Advances }\end{array}$ & 1863.66 & 214.98 & 270.17 & 863.03 & 5289.95 & 4753.82 & 8593.2 & 6835.5 & 4737.45 & 7663.99 \\
\hline Investments & - & 191 & 162.05 & 45.05 & 301.53 & 108.48 & 394.23 & 2079.04 & 2020.3 & 9317.25 \\
\hline $\begin{array}{l}\text { Foreign } \\
\text { Currency Assets }\end{array}$ & 146.54 & 167.77 & 44.58 & 84.74 & 175.01 & 330.03 & 85.08 & 9.23 & - & - \\
\hline Total & 2010.2 & 573.75 & 476.8 & 992.82 & 5766.49 & 5192.33 & 9072.51 & 8923.77 & 6757.75 & 16981.24 \\
\hline $\begin{array}{l}\text { Particulars } \\
2016\end{array}$ & Day 1 & 2-7 days & $\begin{array}{l}8-14 \\
\text { days }\end{array}$ & $\begin{array}{l}15-28 \\
\text { days }\end{array}$ & $\begin{array}{l}29 \text { days } \\
\text { and upto } 3 \\
\text { months }\end{array}$ & $\begin{array}{l}\text { Over } 3 \text { months } \\
\text { and upto } 6 \\
\text { months }\end{array}$ & $\begin{array}{l}\text { Over } 6 \\
\text { months and } \\
\text { upto } 1 \text { year }\end{array}$ & $\begin{array}{l}\text { Over } 1 \text { year } \\
\text { and upto } 3 \\
\text { years }\end{array}$ & $\begin{array}{l}\text { Over } 3 \text { year } \\
\text { and upto } 5 \\
\text { years }\end{array}$ & $\begin{array}{l}\text { Over } 5 \\
\text { years }\end{array}$ \\
\hline $\begin{array}{l}\text { GAP(Assets- } \\
\text { Liabilities) }\end{array}$ & 1696.93 & -360.42 & -390.01 & -310.63 & -4860.96 & -2311.54 & -7935.29 & 2062.15 & 4924.62 & 3883.23 \\
\hline
\end{tabular}

Source: Annual Report, SIB, March, 2016

Maturity Ladder Based on Contractual Maturities 2016:

\begin{tabular}{|c|c|c|c|c|c|c|}
\hline $\begin{array}{l}\text { DAY } 1 \\
\text { CASH INFLOWS }\end{array}$ & AMOUNT & CASH OUTFLOWS & AMOUNT & GAP & RBI TOLERANCE LIMITS & \\
\hline Loans and Advances & 1863.66 & Deposits & 212.74 & & \multirow{4}{*}{$\begin{array}{l}\text { Negative Gap should not be more } \\
\text { than } 5 \% \text { of total outflows }\end{array}$} & \multirow{4}{*}{$\begin{array}{l}\text { Positive gap and RBI } \\
\text { compliant }\end{array}$} \\
\hline Investments & - & Borrowings & 63.69 & & & \\
\hline Foreign Currency Assets & 146.54 & Foreign Currency Liabilities & 36.84 & & & \\
\hline Total & 2010.2 & & 313.27 & 1696.93 & & \\
\hline $\begin{array}{l}\text { DAY 2-7 } \\
\text { CASH INFLOWS }\end{array}$ & & CASH OUTFLOWS & & & \multirow{5}{*}{$\begin{array}{l}\text { Negative Gap should not be more } \\
\text { than } 10 \% \text { of total outflows }\end{array}$} & \multirow{5}{*}{$\begin{array}{l}\text { Negative gap. Not RBI } \\
\text { Compliant }\end{array}$} \\
\hline Loans and Advances & 214.98 & Deposits & 930.69 & & & \\
\hline Investments & 191 & Borrowings & - & & & \\
\hline Foreign Currency Assets & 167.77 & Foreign Currency Liabilities & 3.48 & & & \\
\hline Total & 573.75 & & 934.17 & -360.42 & & \\
\hline $\begin{array}{l}\text { 8-14 days } \\
\text { CASH INFLOWS }\end{array}$ & & CASH OUTFLOWS & & & $\begin{array}{l}\text { Negative Gap should not be more } \\
\text { than } 15 \% \text { of total outflows }\end{array}$ & \\
\hline Loans and Advances & 270.17 & Deposits & 863.88 & & & \\
\hline Loans and Advances & 270.17 & Deposits & 863.88 & & $\begin{array}{l}\text { bank has breached RBI tolerance } \\
\text { limits. }\end{array}$ & $\begin{array}{l}\text { Negative gap and not } \\
\text { RBI Compliant }\end{array}$ \\
\hline Investments & 162.05 & Borrowings & - & & & \\
\hline Foreign Currency Assets & 44.58 & Foreign Currency Liabilities & 2.93 & & & \\
\hline Total & 476.8 & & 866.81 & -390.01 & & \\
\hline $\begin{array}{l}\text { 15-28days } \\
\text { CASH INFLOWS }\end{array}$ & & CASH OUTFLOWS & & & & \\
\hline Loans \& Advances & 863.03 & Deposits & 1290.92 & & \multirow{4}{*}{$\begin{array}{l}\text { Negative Gap should not be more } \\
\text { than } 20 \% \text { of the cash outflows }\end{array}$} & \multirow{4}{*}{ Negative gap } \\
\hline Investments & 45.05 & Borrowings & - & & & \\
\hline Foreign Currency Assets & 84.74 & Foreign Currency Liabilities & 12.53 & & & \\
\hline Total & 992.82 & Total & 1303.45 & -310.63 & & \\
\hline
\end{tabular}




\begin{tabular}{|c|c|c|c|c|c|}
\hline $\begin{array}{l}29 \text { days and upto } 3 \text { months } \\
\text { CASH INFLOWS }\end{array}$ & & CASH OUTFLOWS & & & \\
\hline Loans \& Advances & 5289.95 & Deposits & 8709.88 & & \\
\hline Investments & 301.53 & Borrowings & 927.57 & & \\
\hline Foreign Currency Assets & 175.01 & Foreign Currency Liabilities & 990 & & \\
\hline Total & 5766.49 & Total & 10627.45 & -4860.96 & \\
\hline $\begin{array}{l}\text { Over } 3 \text { months and upto } 6 \\
\text { months } \\
\text { CASH INFLOWS }\end{array}$ & & CASH OUTFLOWS & & & \\
\hline Loans \& Advances & 4753.82 & Deposits & 6860.07 & & \\
\hline Investments & 108.48 & Borrowings & 336.27 & & \\
\hline Foreign Currency Assets & 330.03 & Foreign Currency Liabilities & 307.53 & & \\
\hline Total & 5192.33 & Total & 7503.87 & -2311.54 & Negative gap \\
\hline $\begin{array}{l}\text { Over } 6 \text { months and upto } \\
1 \text { year } \\
\text { CASH INFLOWS }\end{array}$ & & CASH OUTFLOWS & & & Negative gap \\
\hline Loans \& Advances & 8593.2 & Deposits & 16672.03 & & \\
\hline Investments & 394.23 & Borrowings & 170.63 & & \\
\hline Foreign Currency Assets & 85.08 & Foreign Currency Liabilities & 165.14 & & \\
\hline Total & 9072.51 & Total & 17007.8 & -7935.29 & \\
\hline $\begin{array}{l}\text { Over } 1 \text { year and upto } 3 \\
\text { years CASH INFLOWS }\end{array}$ & & CASH OUTFLOWS & & & \\
\hline $\begin{array}{l}\text { Over } 1 \text { year and upto } 3 \\
\text { years CASH INFLOWS }\end{array}$ & & CASH OUTFLOWS & & & Positive gap \\
\hline Loans \& Advances & 6835.5 & Deposits & 6152.9 & & \\
\hline Investments & 2079.04 & Borrowings & 316.79 & & \\
\hline Foreign Currency Assets & 9.23 & Foreign Currency Liabilities & 391.93 & & \\
\hline Total & 8923.77 & Total & 6861.62 & 2062.15 & \\
\hline $\begin{array}{l}\text { Over } 3 \text { year and upto } 5 \\
\text { years } \\
\text { CASH INFLOWS }\end{array}$ & & CASH OUTFLOWS & & & \\
\hline Loans \& Advances & 4737.45 & Deposits & 1229.61 & & Positive gap \\
\hline Investments & 2020.3 & Borrowings & 200 & & \\
\hline Foreign Currency Assets & - & Foreign Currency Liabilities & 403.52 & & \\
\hline Foreign Currency Assets & & Foreign Currency Liabilities & 403.52 & & \\
\hline Total & & Total & 1833.13 & 4924.62 & \\
\hline $\begin{array}{l}\text { Over } 5 \text { years } \\
\text { CASH INFLOWS }\end{array}$ & & CASH OUTFLOWS & & & \\
\hline Loans \& Advances & & Deposits & 12798.01 & & Gap is positive. \\
\hline Investments & & Borrowings & 300 & & \\
\hline Foreign Currency Assets & & Foreign Currency Liabilities & - & & \\
\hline Total & & Total & 13098.01 & 3883.23 & \\
\hline
\end{tabular}

In 2016, on day 1 they have positive gap. In the 2 nd time bucket negative gap should not be more than 10 per cent of total outflows. So it is not RBI Compliant. But on the 2nd time bucket there's a negative gap of 38 percent of the total cash outflows. On the $3^{\text {rd }}$ time bucket there is a negative gap 44 per cent, $4^{\text {th }}$ time bucket there is 23 per cent and so on. On the $7^{\text {th }}$ time bucket onwards they have positive gaps and from that they could keep liquidity. 


\section{Stock Approach:}

The following ratios are calculated from the annual statements of South Indian Bank

The required data for finding these ratios are given below:

Exhibit 7

\begin{tabular}{|l|l|l|l|l|l|l|}
\hline Particular & 2011 & 2012 & 2013 & 2014 & 2015 & 2016 \\
\hline Core deposits & 29721.08 & 36500.53 & 44262.3 & 47491.09 & 51912.49 & 55720.73 \\
\hline Total assets & 29844.47 & 37337.39 & 44846.23 & 51242.84 & 55020.46 & 56747.66 \\
\hline Net loans & 20488.73 & 27280.74 & 31815.53 & 36229.86 & 37391.64 & 41085.75 \\
\hline Short-term liabilities & 15885.03 & 25261.73 & 28388.64 & 30500.3 & 39793.33 & 38556.82 \\
\hline Liquid assets & 63793.5 & 10686.977 & 26392.01 & 10171.21 & 11535.03 & 62334.43 \\
\hline
\end{tabular}

Source: Annual reports on various years

Here we can see that core deposits increase year after year. In case of total assets thus also increase. Net loans increase from 2011-2016. In 2016 we can see that there is an increase in liquid assets. From these data we can find out the assert liability liquidity ratios.

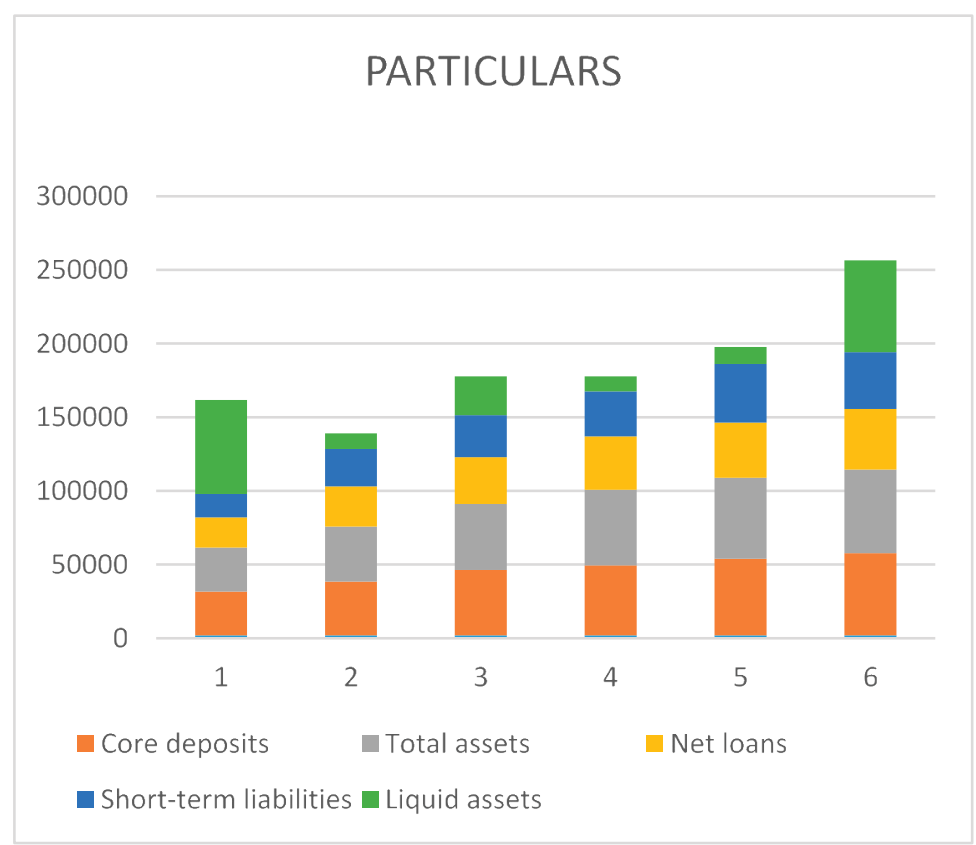

Source: Annual reports on various years

The table below gives as the values of ratios in each year:

\section{Exhibit 8}

\begin{tabular}{|l|l|l|l|l|l|l|}
\hline RATIOS & 2011 & 2012 & 2013 & 2014 & 2015 & 2016 \\
\hline Core Deposits/Total Assets & 0.99586 & 0.97758 & 0.98697 & 0.92678 & 0.94351 & 0.98190 \\
\hline Net Loans/Total Deposits & 0.68936 & 0.74740 & 0.71879 & 0.76287 & 0.72028 & 0.73735 \\
\hline Short term liabilities/Liquid Assets & 0.24900 & 2.3637 & 1.07565 & 2.99869 & 3.4497 & 0.61854 \\
\hline Liquid Assets/Total Assets & 2.13753 & 0.28622 & 0.58850 & 0.19849 & 0.20964 & 1.09844 \\
\hline Short term Liabilities/Total Assets & 0.5322 & 0.6765 & 0.6330 & 0.59521 & 0.72324 & 0.67944 \\
\hline
\end{tabular}

Source: Computed from figures taken from Annual reports of various years. 
- In the case of ratio of Core deposits to Total Assets, more the ratio better it is because core deposits are treated to be stable source of liquidity. In the year 2011, the ratio is 99 percent. In 2016 the ratio is 98 per cent, showing a stability over years.

- Net Loans to Total Deposits Ratio, in the year 2011 is 68 per cent. This ratio is also stable over years.

- Ratio of Short-Term Liabilities to Liquid Assets, is good when the ratio is lower. In the year 2016 it is better for the bank.

- Ratio of Liquid Assets to Total Assets, higher the ratio better it is. We can see that the ratio is higher in the year 2016, so that it will ensure better liquidity.

- Ratio of Short - Term Liabilities to Total Assets here lower ratio is better for the bank. It is to be lower in the interest of liquidity.

\section{Findings}

- In flow approach we can see that there is positive gap as well as negative gap mismatches. It can either be positive or negative. Positive Mismatch: M.A. $>$ M.L. and vice-versa for Negative Mismatch. In case of positive mismatch, excess liquidity can be deployed in money market instruments, creating new assets \& investment swaps etc. For negative mismatch, it can be financed from market borrowings (call/Term), Bills rediscounting, repos \& deployment of foreign currency converted into rupee.

a. In 2011, it was observed that on day 1 there is a positive gap, so it satisfies RBI Norms. On 2-7 days i.e., on $2^{\text {nd }}$ time bucket it is negative it doesn't satisfy RBI Norms, this is similar in the case of next time bucket also. On the $3^{\text {rd }}$ time bucket there's a Negative gap is $33 \%$ of the total cash outflow. Here the bank has breached RBI tolerance limits. On the $4^{\text {th }}$ bucket onwards it's positive gap after that there is a negative gap for that bank should raise the fund from outside to reduce this negative gap. After that they have positive gap on next time buckets.

b. In 2012, on day 1 there is positive gap. After that bank have negative gap i.e., on the $2^{\text {nd }}$ bucket onwards bank possess negative gap of $53 \%$ of total outflow. So it is not RBI Compliant So there is liquidity problem, bank should unable to meet short term financial demands. From $9^{\text {th }}$ time bucket onwards there's positive gap.

c. In 2013 , on $2^{\text {nd }}$ time bucket Negative gap is $50 \%$ of the total cash outflows. So, it is not RBI Compliant here bank faces negative gap, to overcome that they have to raise funds from outside to meet their liabilities. On, the $7^{\text {th }}$ time bucket they have a positive gap, after that there is a negative gap. After this level they maintain positive gap

d. In 2014, on the $1^{\text {st }}$ time bucket they have positive gap and they satisfy RBI Compliant. On $2^{\text {nd }}$ time bucket i.e., 2-7 day there is $53 \%$ of negative gap this can't satisfy RBI Compliant. After this they face liquidity problems to the next time bucket. Then over 6 months and up to 1 year they have positive gap. On the next time bucket they have a negative gap and they cover this gap on the preceding time buckets.

e. In 2015 , on the $1^{\text {st }}$ and $2^{\text {nd }}$ time bucket there is positive gap and bank should satisfy RBI Compliant. On the $3^{\text {rd }}$ time bucket onwards there's a Negative gap of $70 \%$ of the total cash outflows. So it is not upto the RBI Compliant, this arises due to holding a valuable asset that can't be tradable when they need cash during to the lack of buyers. On the $7^{\text {th }}$ time bucket the bank should satisfy RBI Compliant.

f. In 2016, on day 1 they have positive gap. On the $2^{\text {nd }}$ time bucket there's a Negative gap of $38 \%$ of the total cash outflows. So it is not RBI Compliant. In the $2^{\text {nd }}$ time bucket Negative Gap should not be more than $10 \%$ of total outflows. On the $3^{\text {rd }}$ time bucket there's a negative gap 44\%, 4th time bucket there's $23 \%$ and so on. On the $7^{\text {th }}$ time bucket onwards they have positive gaps and from that they should satisfy RBI Compliant.

- In Stock approach it can see that sometimes the ratio is higher and it is lower.

a. In the case of ratio of Core deposits to Total Assets, more the ratio better it is because core deposits are treated to be stable source of liquidity. In the year $2011,99 \%$ is there. In 2016 the ratio is $98 \%$, so it is better compared to other years. 
b. In the case of Net Loans to Total Deposits Ratio, in the year 2011, 68\% is there. This ratio is better when it has lower value.

c. Ratio of Short-Term Liabilities to Total Assets, it is good when the ratio is lower. In the year 2016 it's better for the bank.

d. Ratio of Liquid Assets to Total Assets, higher the ratio better it is. We can see that the ratio is higher in the year 2016, so that it will ensure better liquidity.

e. Ratio of Short - Term Liabilities to Liquid Assets, here lower ratio is better for the bank. It is to be lower in the interest of liquidity.

This study may be a motivation for further studies in the specific areas of liquidity risk management.

\section{Conclusion}

While analysing the liquidity in Flow approach and Stock approach we can see that there is a better liquidity for the South Indian Bank to meet obligations in few time buckets and in cfew time buckets the position needs improvement. The assumptions made in liquidity planning may not be accurate. Besides the assumptions on assets and liabilities and also off-balance sheet items, banks should also anticipate requirement of funds to support other operations like settlements and clearing claims. Net overheads expenses, such as rent and salary are not taken into account as a significant source of cash outflow. Sometimes it will be huge at the time of mass retirement or salary revision. Bank has to strengthen its sinking fund to meet this. The mandatory reserve requirements such as CRR and SLR may change substantially according to monetary control measures exercised by RBI. It can squeeze or expand the liquidity which may result in unpredictable effect on liquidity.

The treasury of a Bank plays a very significant role in liquidity management. Effectiveness of the liquidity risk management depends on the efficiency of front, middle and back offices of the treasury in performing integrated cash flow management. The Asset-Liability Committee plays a big role in liquidity risk management. The mismatch gap may lead to liquidity problem. Liquidity policy prescribes minimum liquidity to be maintained, funding of reserve assets, limits on exposure to money market, contingent funding, interbank committed credit lines etc. But liquidity policy of a Bank cannot be kept constant which requires frequent reviews and revisions.

Premature withdrawal of deposits and pre-closure of loans cannot be prevented even with careful planning leading to liquidity shock. Maturity mismatch may also result in price mismatch or vice-versa hitting banks' profitability. Mounting Non-Performing Assets (NPA) is a real threat for banks. They not only hit the earnings, but also block funds for roll over. They may upset the assumptions on assets mentioned elsewhere. Though the NPA level of South Indian Bank is alarming, it should take adequate steps to meet this challenge in future.

General market conditions are always unpredictable and could affect the liquidity of Bank. The Bank should have a reliable MIS designed to provide timely and forward-looking information on the liquidity position of the bank and the Group to the Board and ALCO, both under normal and stress situations. The MIS should cover liquidity positions in all currencies in which the Bank conducts its business - both on a subsidiary/branch basis (in all countries in which the bank is active) and on an aggregate group basis. It should capture all sources of liquidity risk, including contingent risks and those arising from new activities, and have the ability to furnish more granular and time sensitive information during stress events.

The Management should ensure that an independent party regularly reviews and evaluates the various components of the bank's liquidity risk management process. These reviews should assess the extent to which the bank's liquidity risk management complies with the regulatory/supervisory instructions as well as its own policy. The independent review process should report key issues requiring immediate attention, includ- 
ing instances of non-compliance to various guidances limits for prompt corrective action consistent with the board approved policy. But the challenge is that who should be this 'independent party' and what are their competencies.

The guidelines also cover two minimum global regulatory standards, viz., Liquidity Coverage Ratio (LCR) and Net Stable Funding Ratio (NSFR) as set out in Basel III rules text. But so far RBI Guidelines are silent on these standards. These concepts need clarifications before the South Indian Bank can act.

The "global financial crisis of 2007" has highlighted the role of prudent liquidity management as the cornerstone of financial stability. BCBS has come out with guidelines for liquidity management in banks and Reserve Bank of India has given guidelines after a lot of discussions on November 7, 2012. Though South Indian Bank has already taken adequate steps in this direction, they face a lot of challenges in liquidity risk management. Some of them are discussed in this paper. While following the guidelines meticulously, the Bank should also evolve Contingency Funding Plan (CFP) for meeting the emergency. It is believed that the guidelines on liquidity management will be implemented and stabilized in South Indian Bank soon.

\section{Bibliography}

Bank for International Settlements. (2008). Principles for Sound Liquidity Risk Management and Supervision. Basel Committee on Banking Supervision [online]. Available: crossref.

Ernst \& Young. (2009). Measuring and managing liquidity risk. Online Available: crossref.

SIB students' economic forum. (January 2011). Theme No. 230: New Capital Framework for Banks - Basel III Part 1. SIB students' economic forum. (February 2011.) Theme No. 231: New Capital Framework for Banks - Basel III Part 2. SIB student's economic forum. (February 2012). Theme No. 243: Basel III: RBI's Draft Guidelines and Banks' Preparedness.

SIB student's economic forum. (November 2013). Theme No. 264: Banking Structure In India - The Way Forward. Subramoniam, K. (2015). The IUP Journal of Bank Management. 14(3), 2015.

Shekhar, K. C. Banking Theory and Practice, 20 $0^{\text {th }}$ Edition, Vikas Publication.

Padmalatha, S. \& Justin, P. (2010). Liquidity Risk Management and Supervisory Challenges.

Websites/URLs

- www.rbi.org.in

- www.southindianbank.com (Annual Reports)

- http://www.bis.org/bcbs/basel3/basel3_phase_in_ arrangements.pdf 